\begin{tabular}{|c|l|}
\hline Title & Latitude of Eastward Jet Prematurely Separated from the Western Boundary in a Two-Layer Quasigeostrophic Model \\
\hline Author(s) & Sue, Y asunori; Kubokawa, A tsushi \\
\hline Citation & $\begin{array}{l}\text { Journal of physical oceanography, 45(3), 737-754 } \\
\text { https://doi.org/10.1175/JPO-D-13-058.1 }\end{array}$ \\
\hline Issue Date & 2015-03 \\
\hline Doc URL & http://hdl.handle.net/2115/59852 \\
\hline Type & article \\
\hline File Information & JPO-D-13-058.1.pdf \\
\hline
\end{tabular}

Instructions for use 


\title{
Latitude of Eastward Jet Prematurely Separated from the Western Boundary in a Two-Layer Quasigeostrophic Model
}

\author{
YASUNORI SUE \\ Graduate School of Environmental Science, Hokkaido University, Sapporo, Hokkaido, Japan \\ ATSUSHI KuBOKAwA \\ Faculty of Environmental Earth Science, Hokkaido University, Sapporo, Hokkaido, Japan
}

(Manuscript received 11 March 2013, in final form 7 December 2014)

\begin{abstract}
This paper investigates the formation of eastward jets extended from western boundary currents, using a simple two-layer quasigeostrophic (QG) model forced by a wind stress curl consistent with the formation of a subtropical gyre. The study investigated the dependency of the latitude of the eastward jet on various parameters and on the meridional distribution of the Ekman pumping velocity. The parameters considered in the present study included the viscous and inertial western boundary layer width, the parameter representing the degree of the partial-slip boundary condition, the ratio of the upper- to lower-layer depth, and the bottom friction. With the parameters used, two types of stable structures are found in the time-mean field. One type of structure represented the "prematurely separated jet case," in which the eastward extension jet was located far south of the northern boundary of the subtropical gyre, as is the Kuroshio Extension; the other type was the "gyre boundary jet case," in which the eastward jet occurred along the northern boundary. The initial condition decides which type of structure would occur. When the prematurely separated jet case occurred, the authors found that the latitude of the eastward jet depended very little on the parameters. In addition, this study also observed that the latitude was determined by the meridional distribution of the Ekman pumping velocity. The eastward extension jet was usually located near the latitude that was half of the maximum value of the Sverdrup streamfunction and satisfied an integral condition derived from the QG potential vorticity equation.
\end{abstract}

\section{Introduction}

Until fine-grid eddy-resolving models were available, understanding the separation of western boundary currents and the formation of their eastward extensions was one of the most important subjects in physical oceanography. Prior to the availability of such models, model simulation could not accurately reproduce the western boundary current separation that occurs in oceans, for example, in the case of the separation of the Kuroshio and Gulf Stream. Model western boundary currents showed a tendency to separate far north of the real separation latitude and the separated currents were weaker and broader than reality and flowed eastward along a course

Corresponding author address: Yasunori Sue, Graduate School of Environmental Science, Hokkaido University, Kitaku, Kita 8, Nishi 5, Sapporo, Hokkaido 060-0808, Japan.

E-mail: sue@ees.hokudai.ac.jp near the subtropical/subpolar gyre boundary predicted by the Sverdrup theory (Bryan and Cox 1972; Takano et al. 1973; Han 1984). In the 1990s, eddy-resolving models were developed with improvements in computing power and successfully used to reproduce the Kuroshio and Gulf Stream (Bleck et al. 1995; Smith et al. 2000) and their extensions with inertial recirculation gyres, which suggest the importance of nonlinearity (e.g., Ierley 1990; Kamenkovich et al. 1995). Development of these numerical models of ocean circulation was reviewed in detail by McWilliams (1996).

In spite of the success in reproducing western boundary current separation and eastward extensions, the dynamics of western boundary current separation and the maintenance of eastward jets have not been satisfactorily clarified yet. Because the separation latitude of the Kuroshio coincides with that of the southern coast of Japan and the Gulf Stream separates north of Cape Hatteras, the effects of topographic features of the coastal boundary and/or 
bottom have also been studied (see, e.g., Dengo 1993; Özgökmen et al. 1997; Marshall and Tansley 2001). Recently, Nakano et al. (2008) showed, by using an eddyresolving ocean GCM with no-slip boundary condition, that the model Kuroshio separates around the same latitude and a realistic Kuroshio Extension accompanying the recirculation gyres occurs even when the topography including the Japan Islands is removed. Therefore, the separation of western boundary currents and their eastward extensions can occur even in a rectangular basin (bounded by meridional and zonal coastlines), although the topography must affect the current in real oceans.

A number of studies have been performed to clarify the properties of the circulation structure in a rectangular basin. First, we call to mind the work of Haidvogel et al. (1992). They investigated the dependence of the separation latitude on the wall boundary condition using a partial-slip boundary condition and biharmonic viscosity. The authors showed that western boundary currents separate far upstream from the gyre boundary predicted by Sverdrup theory ("premature separation" in their terminology), when the applied western boundary condition is close to a no-slip condition using a three-layer quasigeostrophic (QG) model. Although the appropriate, specific boundary condition for defining the real western boundary is not clear, shear with opposite sign on the coastal side of the western boundary current to that on the offshore side seems necessary for premature separation to occur in numerical models, as mentioned by Dengo (1993) and Kiss (2002), and the no-slip boundary condition at the boundary could be regarded as a parameterization necessary to create the shear layer.

Verron and Blayo (1996) investigated the effects of the scheme estimating the vorticity at the no-slip boundary on the separation problem using a three-layer QG model. They showed that the scheme can strongly affect the circulation pattern when the viscous boundary layer is thinner than the grid size. It was also shown that the asymmetry of wind stress curl affects the separation point (see also Verron and Le Provost 1991). Dynamical system approaches have also been used to investigate structures of large-scale ocean circulation under nonlinear conditions and their dependence on parameters relating to nonlinearity and viscosity. Verron and Jo (1994) investigated the solution types of the circulation and classified them as stable, unstable, or intermediate solutions in a broad range of parameters of viscous and inertial boundary layer width. While Ierley and Sheremet (1995) found multiple solutions at the same parameter values in a barotropic single gyre, Cessi and Ierley (1995) studied symmetry breaking caused by the pitchfork bifurcation for a double-gyre circulation. Further increase in nonlinearity causes a Hopf bifurcation that yields periodic solutions (Jiang et al. 1995). Although the asymmetric solutions found in those articles would be related to the problem of the western boundary current separation, the solutions were very different from that with premature separation obtained by Haidvogel et al. (1992) in which the separation occurs in the subtropical and subpolar gyres symmetrically. The difference could partly arise from the differences in the parameter values and also from the model vertical structure; the models used in the above dynamical system approach were barotropic or 1.5-layer models, while Haidvogel et al. (1992) used a three-layer model in a more turbulent regime.

Many recent studies have addressed the dynamics and variability of real western boundary currents and their influences on climate, marine ecosystems, and so on. Sasaki and Schneider (2011) studied the mechanism of meridional shifts of the Kuroshio Extension jet that propagates from east to west. Sugimoto and Hanawa (2012) showed that the Kuroshio path on the southern coast of Japan is correlated with the stability of the Kuroshio Extension jet. Frankignoul et al. (2011) studied the influence of the meridional shifts of the KuroshioOyashio extensions on the atmospheric circulation, and Sasai et al. (2010) studied the effects of variability of the Kuroshio Extension on the marine ecosystem, for example. However, it seems that one of the most basic questions still remains unanswered: what are the parameters that govern the pattern of wind-driven circulation in a rectangular basin, and what determines the latitude of the prematurely separated eastward jet? To address these questions, this paper revisits the problem of separation of a western boundary current and formation of an eastward jet in a two-layer QG model, which is one of the simplest models able to produce a premature separation. Premature separation could also be seen in a barotropic sliced cylinder model in Griffiths and Kiss (1999) and Kiss (2002) with a horizontally uniform surface stress curl and a curved boundary. We will show later in the article that two active layers are needed for premature separation to occur in our rectangular basin model. Based on the parameter dependence of the circulation pattern, we will also discuss the dynamics determining the latitude of the extended jet.

After describing the model configuration in section 2, we will show typical circulation patterns in the present model and discuss the dependence of the latitude of the eastward extension jet on the parameters and external forcing in section 3. Section 4 discusses the factors that determine the latitude of the jet after separation from the western boundary, implied by the dependence on the parameters and external forcing. The remainder of that section is reserved for conclusions and remarks. 
TABLE 1. Dimensional parameters.

\begin{tabular}{|c|c|c|}
\hline Parameter & Meaning & Values or definition \\
\hline$\rho_{0}$ & Density of the upper layer & $1000\left(\mathrm{~kg} \mathrm{~m}^{-3}\right)$ \\
\hline$\Delta \rho$ & Density difference between the two layers & $2\left(\mathrm{~kg} \mathrm{~m}^{-3}\right)$ \\
\hline$g$ & Gravitational acceleration & $9.8\left(\mathrm{~m} \mathrm{~s}^{-2}\right)$ \\
\hline$f$ & Coriolis frequency & $f_{0}+\beta y$ \\
\hline$f_{0}$ & Coriolis frequency at the reference latitude & $8.3 \times 10^{-5}\left(\mathrm{~s}^{-1}\right)$ \\
\hline$\beta$ & Meridional gradient of $f$ & $2 \times 10^{-11}\left(\mathrm{~m}^{-1} \mathrm{~s}^{-1}\right)$ \\
\hline$H_{j}$ & Thickness of the $j$ th layer & $\left(H_{1}, H_{2}\right) \approx(1000,4000)(\mathrm{m})$ \\
\hline$H_{0}$ & Whole layer thickness & $H_{0}=H_{1}+H_{2}$ \\
\hline$W_{e}$ & Ekman pumping velocity scale & $U=f_{0} W_{e} / \beta H_{1}$ \\
\hline$R_{I}$ & Rossby radius of deformation & $\sqrt{\frac{\Delta \rho g H_{1} H_{2}}{\rho_{0} f_{0}^{2}\left(H_{1}+H_{2}\right)}} \approx 45(\mathrm{~km})$ \\
\hline$L$ & Meridional basin scale & $F_{I}^{1 / 2} R_{I} \approx 2025(\mathrm{~km})$ \\
\hline$U$ & Velocity scale of the Sverdrup flow & $U=f_{0} W_{e} / \beta H_{1}$ \\
\hline$T$ & Time scale & $1 / \beta L \approx 0.29$ (day) \\
\hline$\nu$ & Coefficient of horizontal viscosity & $\approx 50\left(\mathrm{~m}^{2} \mathrm{~s}^{-1}\right)$ \\
\hline$r$ & Coefficient of bottom Ekman friction & $\approx 4.0 \times 10^{-8}\left(\mathrm{~s}^{-1}\right)$ \\
\hline$\Delta x, \Delta y$ & Grid size & $L / 256 \approx 8.0(\mathrm{~km})$ \\
\hline
\end{tabular}

\section{Model}

We used a two-layer rigid-lid quasigeostrophic model on a $\beta$ plane. The nondimensionalized equations can be written as

$$
\frac{\partial q_{j}}{\partial t}+\frac{1}{\hat{\beta}} J\left(\psi_{j}, q_{j}\right)=(2-j) w_{e}+\hat{\nu} \nabla^{4} \psi_{j}-(j-1) \hat{r} \nabla^{2} \psi_{j},
$$

with

$$
q_{j}=\nabla^{2} \psi_{j}-F_{j}\left(\psi_{j}-\psi_{3-j}\right)+\hat{\beta} y,
$$

where $j$ is the layer number $(j=1$ and $j=2$ denote upper and lower layer, respectively); $t$ is the time; $x$ and $y$ are the eastward and northward coordinates, respectively; $J$ is the Jacobian; and $\nabla$ is the horizontal differential operator. The terms $\psi_{j}$ and $q_{j}$ are the streamfunction and the potential vorticity in the $j$ th layer, respectively. The nondimensionalization was carried out using the meridional length scale of the wind-driven gyre $L$, meridional derivative of the Coriolis parameter $\beta$, and Sverdrup velocity $U$. The time was scaled by $(\beta L)^{-1}$. Tables 1 and 2 list the meaning and the definition of the coefficients along with the dimensional and nondimensional parameters we used. Although the parameters in Eqs. (1) and (2) are $\hat{\beta}, \hat{\nu}, F_{j}$, and $\hat{r}$, we will use the width of the inertial boundary layer divided by the Rossby radius of deformation $R_{I}, \delta_{I}$, that of the viscous boundary layer $\delta_{M}$, the ratio of the upper- to the lower-layer thickness $H_{1} / H_{2}$, and $F_{I}$ whose square root is the meridional length of the model domain divided by the Rossby radius of deformation instead of $\hat{\beta}, \hat{\nu}$, and $F_{j}$. In terms of $\delta_{I}, \delta_{M}, F_{I}$, and $H_{j}$, the parameters $\hat{\beta}, \hat{\nu}$, and $F_{j}$ can be written as

$$
\hat{\beta}=\frac{2 F_{I}}{\delta_{I}^{2}}, \quad \hat{\nu}=\frac{\delta_{M}^{3}}{F_{I}^{3 / 2}}, \quad F_{j}=F_{I} \frac{H_{3-j}}{H_{1}+H_{2}} .
$$

The term $w_{e}$ on the right-hand side of Eq. (1) is the Ekman pumping velocity, which is proportional to the wind stress curl. The Sverdrup balance in Eq. (1) is $\partial \psi_{\mathrm{Sv}} / \partial x=w_{e}$, where $\psi_{\mathrm{Sv}}$ is the Sverdrup streamfunction and subscript $\mathrm{Sv}$ denotes the value estimated by the

TABLE 2. Nondimensional parameters. The bold font in the right column denotes the commonly used value for that parameter.

\begin{tabular}{lll}
\hline \multicolumn{1}{c}{ Parameter } & \multicolumn{1}{c}{ Meaning } & \multicolumn{1}{c}{ Values or definition } \\
\hline$F_{I}^{1 / 2}=L / R_{I}$ & Lateral basin scale in units of $R_{I}$ & $\mathbf{4 5 , 3 0}, 30.22,0.24,0.26,0.28, \mathbf{0 . 3}$ \\
$\delta_{M}=(\nu / \beta)^{1 / 3} R_{I}^{-1}$ & Viscous WBC width in units of $R_{I}$ & $0.2,0.22,0.82, \mathbf{0 . 9}, 0.98,1.06$ \\
$\delta_{I}=\sqrt{2 U / \beta} R_{I}^{-1}$ & Inertial WBC width in units of $R_{I}$ & from 14 to $\mathbf{5 . 7} \times \mathbf{1 0}$ \\
$\alpha$ & Partial-slip coefficient in units of $1 / R_{I}$ & $\frac{1}{1}, \frac{1}{2}, \frac{\mathbf{1}}{\mathbf{4}}, \frac{1}{8}, \frac{1}{16}$ \\
$H_{1}$ & Ratio of first- to second-layer thickness & $0,10^{-4}, \mathbf{1 0}^{-\mathbf{3}}, 10^{-2}$ \\
$H_{2}$ & Coefficient of bottom friction & $2 F_{I} / \delta_{I}^{2}$ \\
$\hat{r}=r / \beta L$ & Nondimensional beta & $\delta_{M}^{3} / F_{I}^{3 / 2}$ \\
$\hat{\boldsymbol{\nu}}$ & Coefficient of horizontal viscosity \\
\hline
\end{tabular}


Sverdrup balance, and $w_{e}(x, y)$ is set so that the maximum of $\psi_{\mathrm{Sv}}$ at the western boundary is unity. That is,

$$
\left[\psi_{\mathrm{Sv}}\left(X_{W}, y\right)\right]_{\max }=\left[\int_{X_{E}}^{X_{W}} w_{e} d x\right]_{\max }=1,
$$

where $X_{E}$ and $X_{W}$ are the $x$ coordinates of the eastern and western boundaries, respectively; we define $Y_{N}$ and $Y_{S}$ as the $y$ coordinates of the northern and southern boundaries in the same way.

The lateral boundary conditions are no-normal flow and partial slip, that is,

$$
\psi_{j}=0
$$

and

$$
\frac{\partial^{2} \psi_{j}}{\partial \eta^{2}}=-\alpha F_{I}^{1 / 2} \frac{\partial \psi_{j}}{\partial \eta}
$$

where $\eta$ is the outward coordinate normal to the boundary, and $\alpha$ is the partial-slip coefficient normalized by the Rossby radius of deformation. The partial-slip boundary condition is regarded as the free-slip condition at $\alpha=0$ and the no-slip condition at the limit of $\alpha \rightarrow \infty$. In the present study, the slip boundary condition was used along the southern, northern, and eastern boundaries, and the partial-slip boundary condition was used along the western boundary. When we wished to adopt the noslip condition, $\alpha$ was set to $5.7 \times 10^{2}$. Although most of the experiments were conducted in the no-slip boundary condition, experiments with various $\alpha$ were also conducted. Based on the above, the parameters governing the present model are

$$
F_{I}, \frac{H_{1}}{H_{2}}, \delta_{I}, \delta_{M}, \hat{r} \text { and } \alpha .
$$

In the present paper, we set $F_{I}^{1 / 2}=45$. To examine the dependence of the solution on the basin scale, we also conducted an experiment in which $F_{I}^{1 / 2}=30$ and found that the circulation pattern showed little difference, which suggests that the dependence on $F_{I}$ is low when the ocean is much larger than the deformation radius.

The Ekman pumping velocity is set to be of the form

$$
w_{e}(x, y)= \begin{cases}\frac{1}{X_{E}-X_{I}} \hat{w}_{e}(y), & \text { for } \quad X_{I} \leq x \leq X_{E}, \\ 0, & \text { for } \quad X_{W}<x<X_{I},\end{cases}
$$

where the maximum value of $\hat{w}_{e}(y)$ is unity. Although $\hat{w}_{e}=\sin \pi y$ is used most often, we used other forms of $\hat{w}_{e}(y)$ to demonstrate the dependence of the solution on the wind stress distribution. In most of the experiments, we set $X_{E}=2, X_{I}=1$, and $X_{W}=0$. In this profile, the western half of the basin was not forced by Ekman pumping. The extended jet meandered vigorously just after separating from the western boundary, and the Ekman pumping velocity tends to weaken the eastward jet (as shown later). The above setting made the extended jet longer in the zonal direction and permitted determination of the spatial mean latitude of the extended jet more unambiguously, which served our purpose.

Before proceeding to the numerical results, we considered typical values of parameters. The dimensional parameters are listed in Table 1 , and the reference value of $H_{1}=1000 \mathrm{~m}$ and $H_{2}=4000 \mathrm{~m}$ gave $R_{I} \simeq 45 \mathrm{~km}$. Based on the above parameter values, the dimensional meridional basin scale $L$ is $2025 \mathrm{~km}$, and the time scale of damping by reference bottom friction, which is $\hat{r}=10^{-3}$, is 290 days. Because in the North Pacific the north-south extent of the subtropical gyre is approximately $2500 \mathrm{~km}$ and the Sverdrup transport is approximately 40 Sverdrups $\left(\mathrm{Sv} ; 1 \mathrm{~Sv} \equiv 10^{6} \mathrm{~m}^{3} \mathrm{~s}^{-1}\right.$ ), we estimated that the westward velocity near the western boundary is approximately $40 \times 10^{6} \mathrm{~m}^{3} \mathrm{~s}^{-1} /(1000 \mathrm{~m} \times 1250 \mathrm{~km}) \simeq 0.036 \mathrm{~m} \mathrm{~s}^{-1}$. This yields an inertial boundary layer with a width of $\sqrt{3.6 \times 10^{-2} / \beta} \simeq 40 \mathrm{~km}$. Therefore, $\delta_{I}$ is approximately 0.9 . Although the width of the viscous boundary layer in the real ocean is unknown, we may assume that it is less than the inertial boundary layer width. In the numerical experiment of Nakano et al. (2008), $\delta_{M}$ was approximately 0.4 , but they used biharmonic viscosity. In the present paper, $\delta_{M}=0.3$ was used as the reference value that corresponded to $\nu=50\left(\mathrm{~m}^{2} \mathrm{~s}^{-1}\right)$. Ierley and Ruehr (1986) showed that for steady broad eastward flow from a nonlinear Munk layer to exist, a parameter $\lambda=$ $\left(\delta_{I} / \delta_{M}\right)^{2} \hat{U}$ is smaller than about 0.8 , where $\hat{U}$ is the eastward velocity in the interior. Since $\left(\delta_{I} / \delta_{M}\right)^{2}$ in our typical case was nearly 10 , it was expected that the condition could not be satisfied almost anywhere; hence, the eastward flow is expected to be a narrow jet accompanying recirculation gyres (Ierley 1987).

We used the conservative form of the interpolated differential operator (IDO-CF) (Imai et al. 2008) as our numerical calculation scheme. This scheme provides a highly accurate solution by solving the equations of cell-integrated values as an interpolation function at the same time, and it also solves the original equations. The grid size, $\Delta x$ and $\Delta y$ for the $x$ direction and $y$ direction, respectively, was set to $1 / 256$ to coincide with the minimum viscous boundary layer width in our experiments. Because the cell-integrated values are also calculated, the assumed grid size is regarded as half the size of $\Delta x$ and $\Delta y$. For several cases, numerical experiments were 
(a)

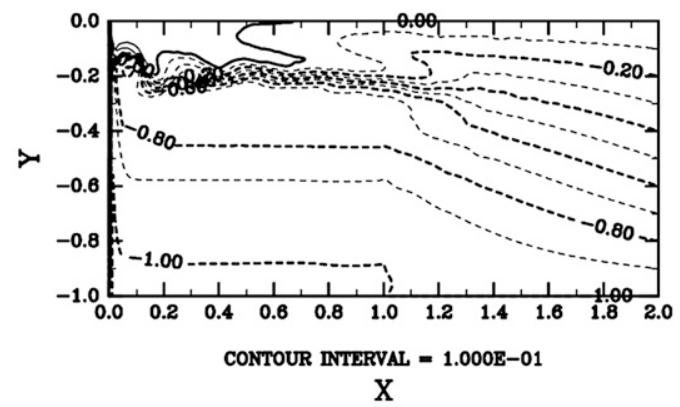

(c)

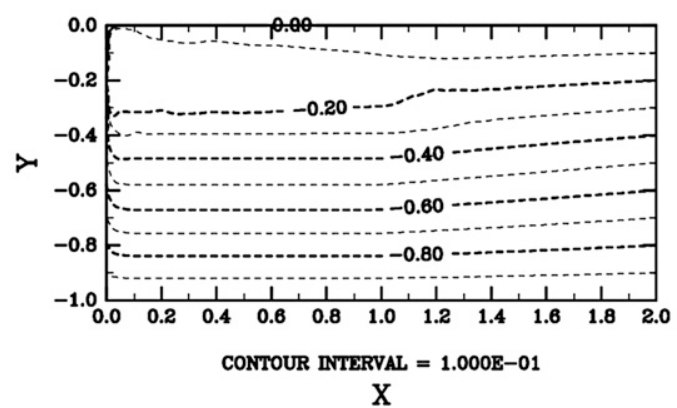

(e)

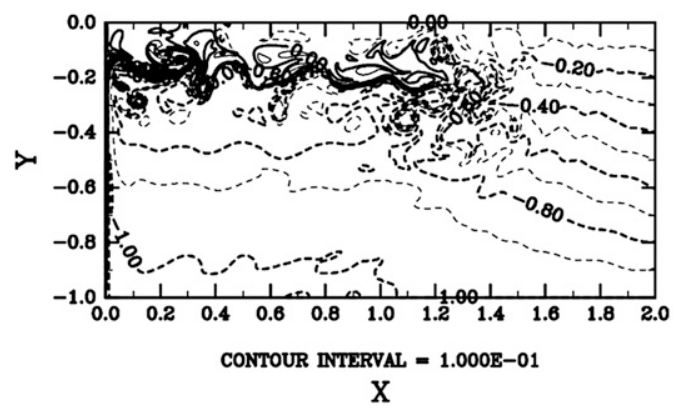

(b)

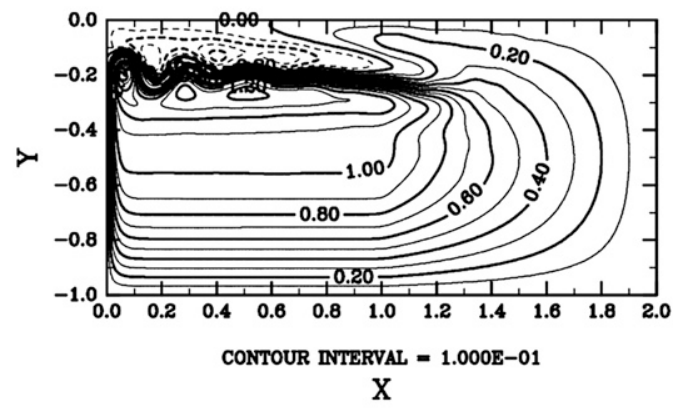

(d)

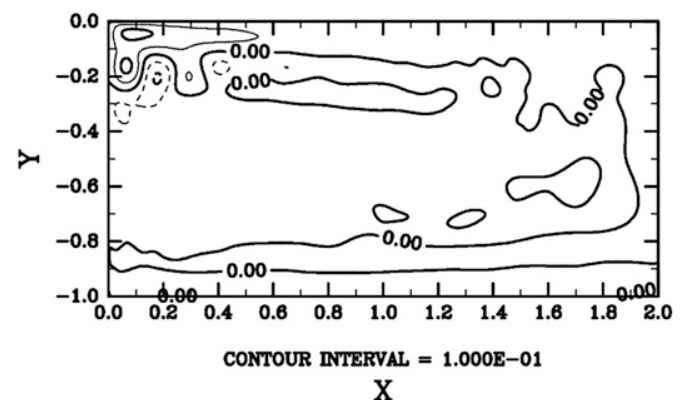

(f)

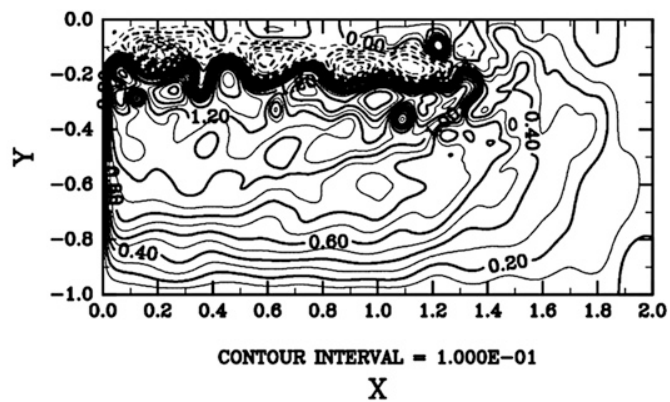

$\mathrm{X}$

FIG. 1. Typical solution of the prematurely separated jet case $\left(\delta_{M}=0.3, \delta_{I}=0.9, H_{1} / H_{2}=1 / 4\right)$ under single-gyre forcing. Temporal-mean fields of the upper-layer potential vorticity divided by (a) $\hat{\beta}$ and (b) streamfunction. (c),(d) As in (a) and (b), but for the lower layer. (e),(f) As in (a) and (b), but for snapshots ( $\mathrm{t}=100000)$ in the upper layer.

conducted using the grid size of $1 / 512$ to confirm that there was no significant difference.

Although we conducted most experiments with singlegyre forcing $\left(Y_{S}=-1\right.$ and $\left.Y_{N}=0\right)$, which produced a subtropical circulation, we assumed an antisymmetric subarctic circulation on the outside of the northern boundary using the slip boundary condition. Therefore, mass conservation was satisfied automatically. The next section describes the differences among experiments in double-gyre $\left(Y_{S}=-1\right.$ and $\left.Y_{N}=1\right)$ as well as in single-gyre conditions. From the initial state with no flow in the upper and lower layers, time integration was conducted over a range greater than 100000 nondimensional times $(80 \mathrm{yr})$, which is 25 times longer than the baroclinic Rossby wave response time. A quasi-equilibrium state was established after approximately 20000 nondimensional times.

\section{Results}

\section{a. Two typical solutions}

Figures 1 and 2 show the streamfunctions and potential vorticity fields of results for $\hat{w}_{e}=\sin \pi y$ with the meridional extent of $-1<y<0$ for two typical solutions. One solution was characterized by an eastward jet that separated prematurely from the western boundary (Fig. 1), and the other was characterized by an eastward jet along the northern boundary, which was regarded as the subtropical/subpolar gyre boundary (Fig. 2). 
(a)

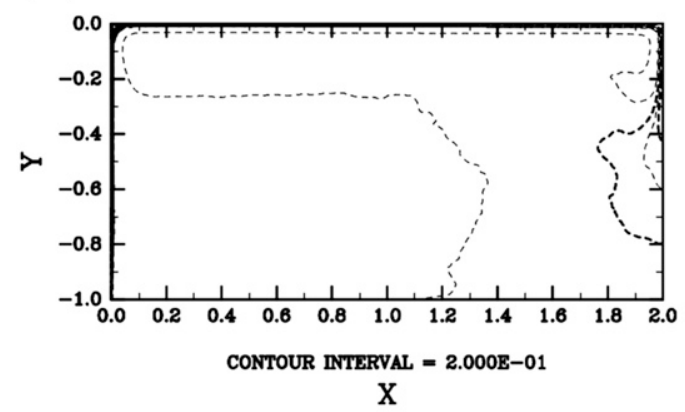

(c)

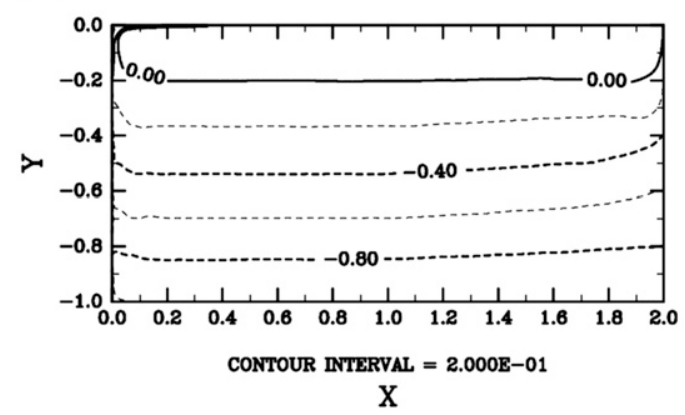

(e)

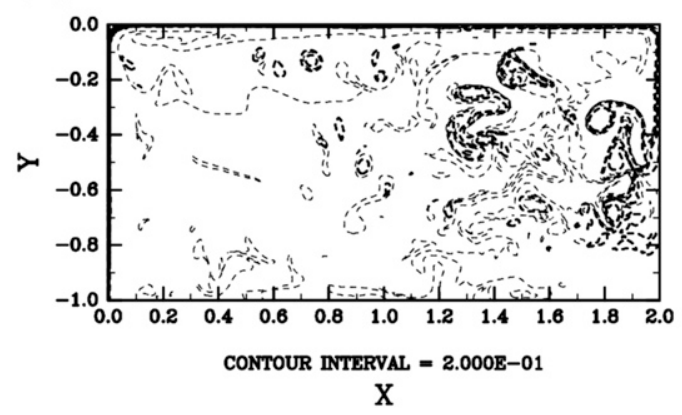

(b)

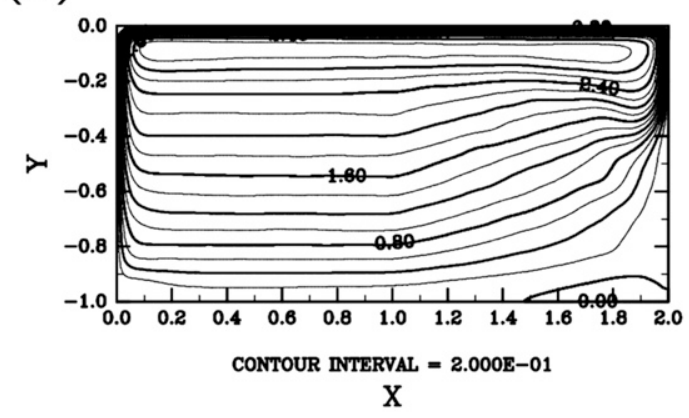

(d)

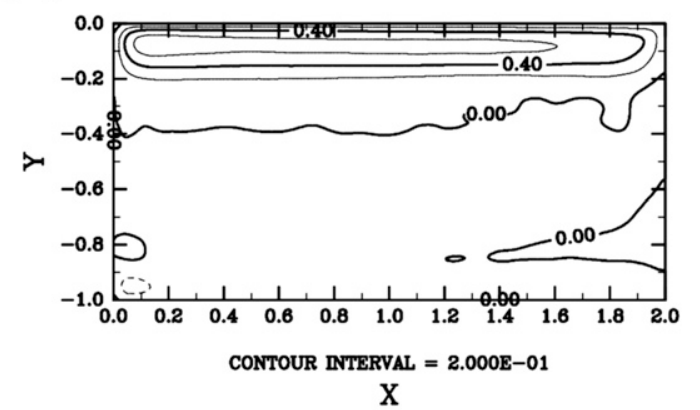

(f)

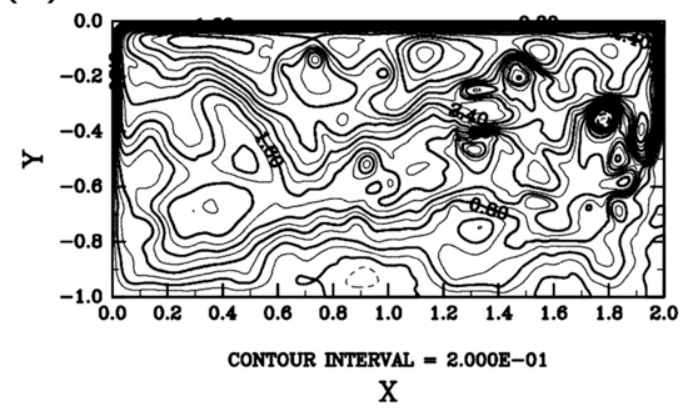

FIG. 2. As in Fig. 1, but for the gyre boundary jet case $\left(\delta_{M}=0.22, \delta_{I}=0.9\right)$.

Although these solutions are very different, the parameter values differ only for $\delta_{M}\left(\delta_{M}=0.3\right.$ in the former case and 0.22 in the latter case); the other parameters are common $\left(\delta_{I}=0.9, H_{1} / H_{2}=1 / 4, \hat{r}=10^{-3}\right.$, and $\left.\alpha=5.7 \times 10^{2}\right)$. We refer to the former solution as the "prematurely separated jet case" and the latter as the "gyre boundary jet case." The latter naming derives from experiments in the double-gyre case. The difference between single- and double-gyre cases will be described below.

In the prematurely separated jet case, the western boundary current separated from the boundary, and the strong eastward extended jet was formed at a latitude of approximately -0.2 , accompanied by northern and southern recirculation gyres on both sides. Although there are many eddies in the snapshots (lower panels in
Fig. 1), we can clearly find a meandering narrow jet. Thus, the structure of the time-mean jet can be substantial. In Fig. 1, the jet is located along the front of low potential vorticity water advected by the inertial boundary current and high potential vorticity water generated by the viscous boundary layer as in Kiss (2002). Northern and southern recirculation gyres in the flank of the jet, mentioned in Nakano et al. (2008), can also be seen in both the upper and lower layers, which imply that these recirculations create strong barotropic structure. The velocity of the extended jet is rapidly damped for $x>1.0$, where the ocean is forced by the Ekman pumping velocity, and it is smoothly connected to the interior Sverdrup flow region. The jet length of the time-mean field, defined as the distance from the western boundary to the westernmost position, where the maximum velocity falls below the half 
(a)

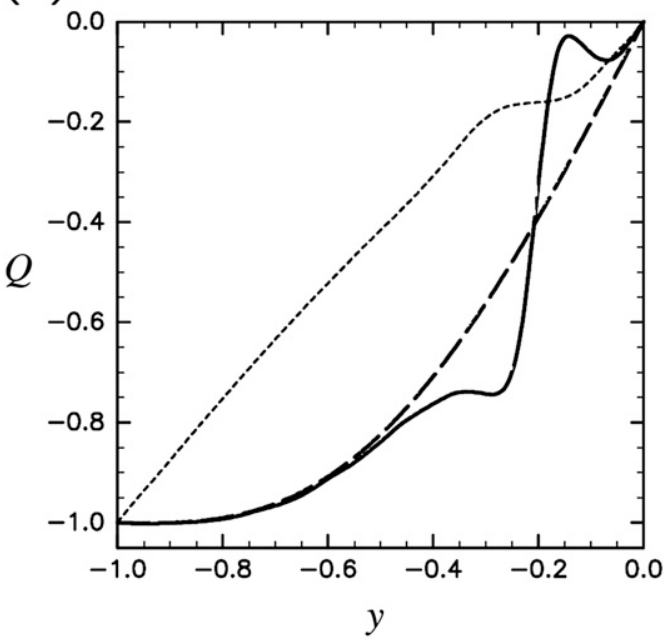

(c)

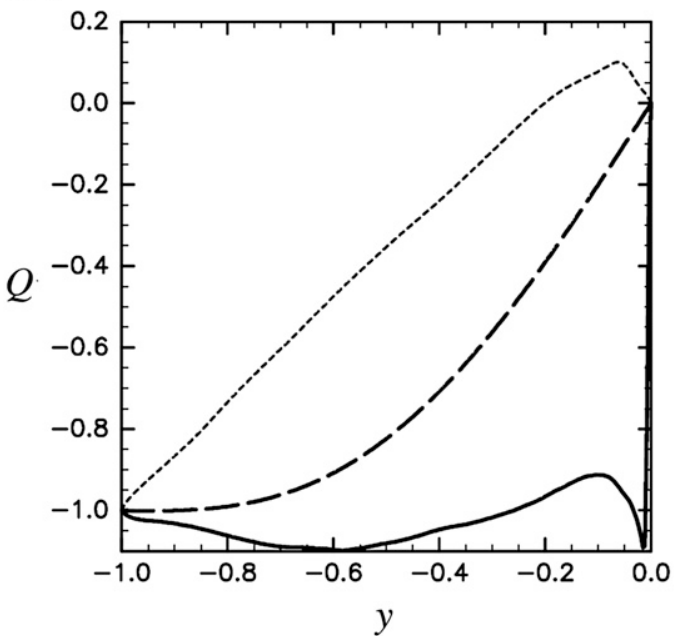

(b)

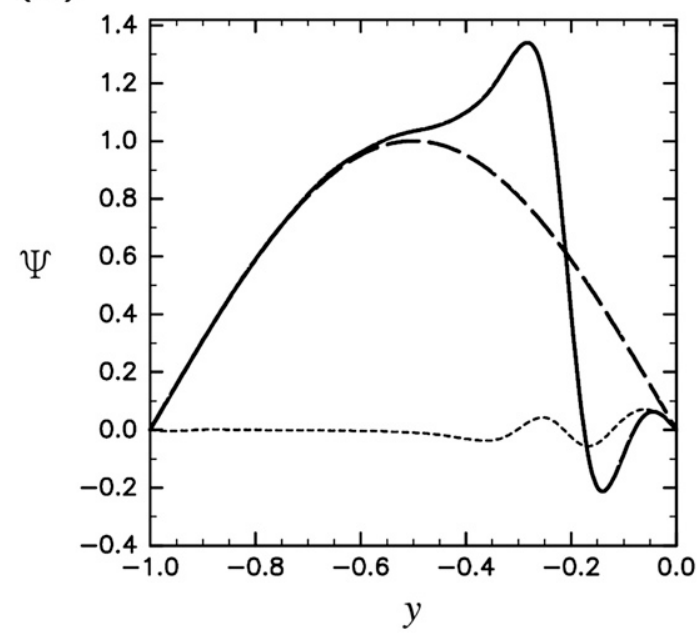

(d)

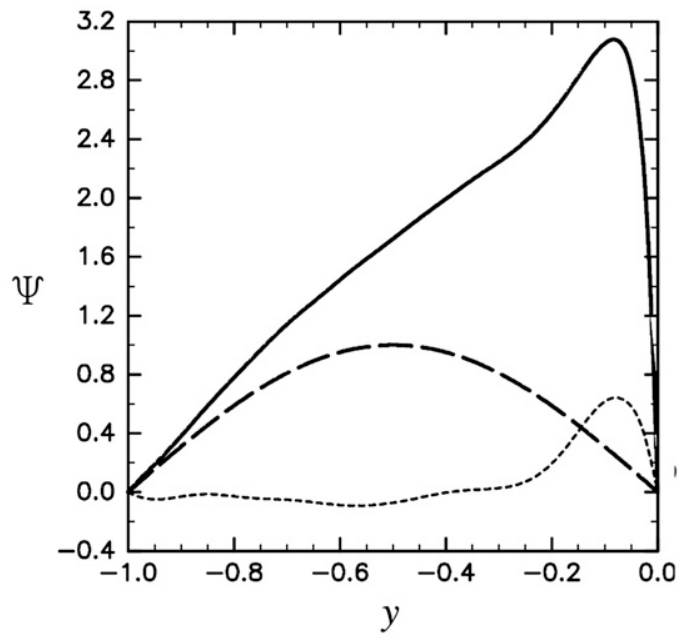

FIG. 3. Meridional profiles of mean potential vorticity divided by (left) $\hat{\beta}$ and (right) mean streamfunction, averaged from $x=0.5$ to $x=1.0$ and in time. (a),(b) The prematurely separated jet case $\left(\delta_{M}=0.30\right.$ and $\left.\delta_{I}=0.9\right)$ and (c),(d) the gyre boundary jet case $\left(\delta_{M}=0.22\right.$ and $\left.\delta_{I}=0.9\right)$ with other parameters as in Fig. 1 . The solid curves are the profiles in the upper layer, the dotted curves are those in the lower layer, and the dashed curves are those estimated by the Sverdrup balance in the upper layer.

of the averaged velocity of the jet from $x=0.5$ to $x=1.0$, is approximately 1.1. Note that the eastern edge of the jet exhibits such large fluctuations that the zonal jet seen in snapshots extends longer than that in the time-mean field. Upper panels in Fig. 3 show the meridional profiles of potential vorticity (left) divided by $\hat{\beta}, Q_{j}(y)$, and the streamfunction (right), $\Psi_{j}(y)$, both of which are averaged from $x=0.5$ to $x=1.0$ for the time mean. The potential vorticity tends to be homogenized at the northern and southern sides of the jet in the upper layer and below the jet in the lower layer. In the southern half region, $\Psi_{1}(y)$ and $Q_{1}(y)$ are consistent with the values estimated by Sverdrup balance:

$$
\begin{aligned}
& \hat{\psi}_{\mathrm{Sv}}(y)=-\hat{w}_{e}(y) \text { and } \\
& \hat{q}_{\mathrm{Sv}}(y)=\frac{1}{\hat{\beta}}\left(\frac{\partial^{2} \hat{\psi}_{\mathrm{Sv}}}{\partial y^{2}}-F_{I} \frac{H_{2}}{H_{0}} \hat{\psi}_{\mathrm{Sv}}\right)+y,
\end{aligned}
$$

where $\hat{\psi}_{\mathrm{Sv}}(y)$ and $\hat{q}_{\mathrm{Sv}}(y)$ are $\psi_{\mathrm{Sv}}$ and $q_{\mathrm{Sv}}$ in the western part of the basin.

In the gyre boundary jet case, the jet is located at the northern boundary, and there is extensive and robust anticyclonic recirculation on its southern side (Fig. 2). The jet reaches the eastern boundary, which is much further than in the prematurely separated jet case. The upper-layer potential vorticity is almost homogenized at 
(a)

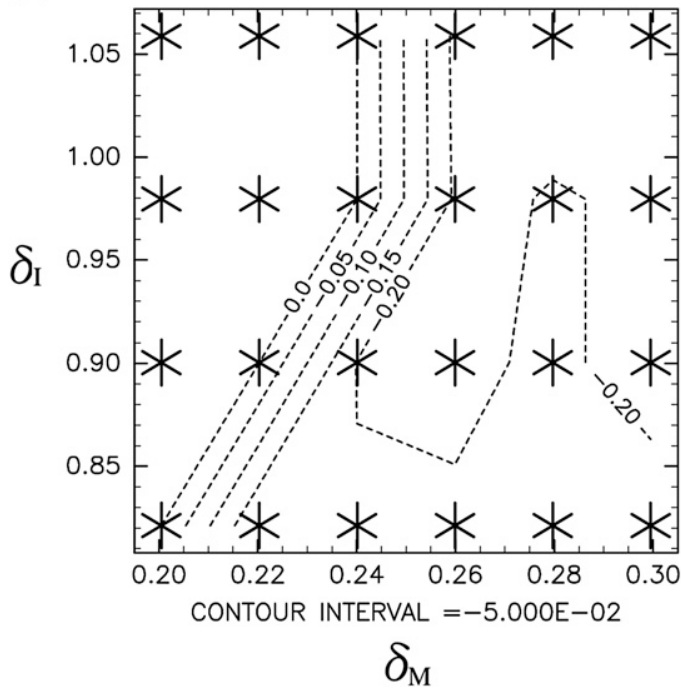

(b)

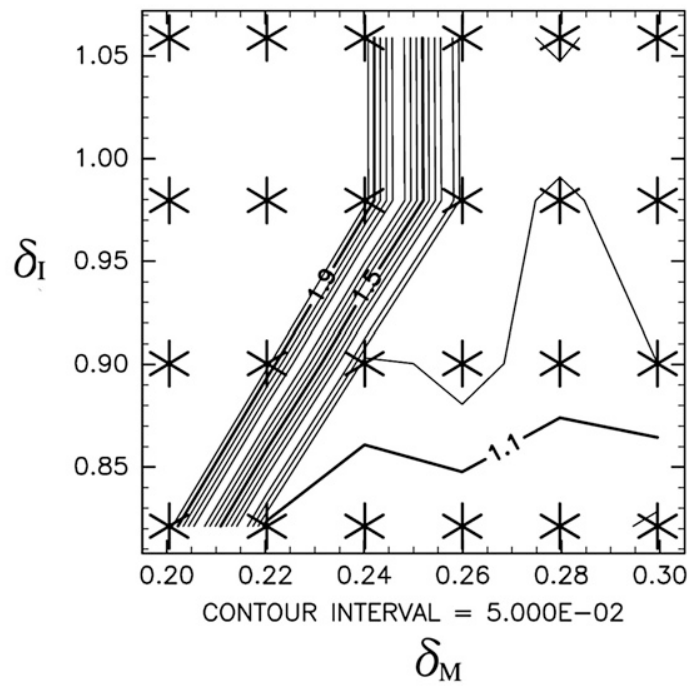

FIG. 4. (a) Dependence of the $y$ coordinate of the eastward extension jet $Y_{\text {Jet }}$ on $\delta_{M}$ and $\delta_{I}$. (b) Dependence of the jet length on $\delta_{M}$ and $\delta_{I}$. The asterisks denote data points for which computations were made. The term $Y_{\text {Jet }}$ changes discontinuously from 0 (gyre boundary jet case) to -0.2 (prematurely separated jet case) between two adjacent computational points, and there is no intermediate value of $Y_{\mathrm{Jet}}$. All experiments were run from rest. The final state depends on the initial condition; in many cases an initial condition in the gyre or prematurely separated jet case will persist even under parameter values in which the other state is found when spun up from rest (see text for details).

that of the southern boundary $\left(Q_{1} \approx-1\right)$, and the lowerlayer vorticity is very small; $\psi_{1} \approx\left[\left(H_{0} \hat{\beta}\right) /\left(F_{I} H_{2}\right)\right] y$ and $\psi_{2} \approx 0$, except near the northern boundary where a barotropic recirculation occurs.

Next, we compared the results obtained using doublegyre forcing with single-gyre forcing cases because double-gyre experiments are much more commonly used for studying basic ocean dynamics, as attempted in this study. In prematurely separated jet cases, there is little difference between a single-gyre case and a double-gyre case in the subtropical region; the meridional profiles of the streamfunction and the potential vorticity are also almost the same (not shown). In the gyre boundary jet case with double-gyre forcing, the eastward jet length was approximately 1.3, much shorter than that for single-gyre cases, and the homogenization of the potential vorticity occurred near the jet and $Q_{j}(y)$ and $\Psi_{j}(y)$ matched $\hat{q}_{\mathrm{Sv}}(y)$ and $\hat{\psi}_{\mathrm{Sv}}(y)$ in the region far from the jet (not shown). Under the conditions of double-gyre forcing, shear instability occurs on the gyre boundary jet, and the jet length is reduced. As a result, the strength of the circulation with single-gyre forcing is quite large relative to the double-gyre case because there is no lateral vorticity mixing through the northern boundary, which was mentioned by Fox-Kemper (2005) in a barotropic model.

Although there are many differences between singleand double-gyre forcing in the gyre boundary jet case, the prematurely separated jet case is more important because it seems to correspond to realistic eastward jets, where single- and double-gyre forcing yield almost the same results; the classification of the solution type for the parameters does not change either. We were in terested in the dependence of the jet separation latitude on parameters. Therefore, we examined the parameter dependence of the solution using single-gyre forcing with $Y_{S}=-1$ and $Y_{N}=0$ to save computing resources.

\section{b. Dependence on parameters}

\section{1) DEPENDENCIES ON VISCOUS AND INERTIAL BOUNDARY LAYER WIDTH}

The dependence of the latitude of the eastward extended jet on $\delta_{I}$ and on $\delta_{M}$ in the temporal mean field was investigated. The latitude of the jet, $Y_{\text {Jet }}$, was defined as the $y$ value at which the maximum time-mean zonal velocity zonally averaged from $x=0.5$ to $x=1$ occurred. The parameter ranged from 0.2 to 0.3 for $\delta_{M}$ and from 0.82 to 1.06 for $\delta_{I}$. The experiment was also conducted under $\delta_{M}=0.4$, which was hardly different from the case in which $\delta_{M}=0.3$. Because dimensional velocities in the model are proportional to $\delta_{I}^{2}$, the set range of $\delta_{I}$ corresponds to 0.8 to 1.4 times the strength of the flow of the typical case in which $\delta_{I}=0.9$.

Figure $4 \mathrm{a}$ shows the latitude of the jet in the $\delta_{M}-\delta_{I}$ plane. When $\delta_{I}$ is larger and $\delta_{M}$ is smaller, the gyre boundary jet case tends to occur. In the case of premature 

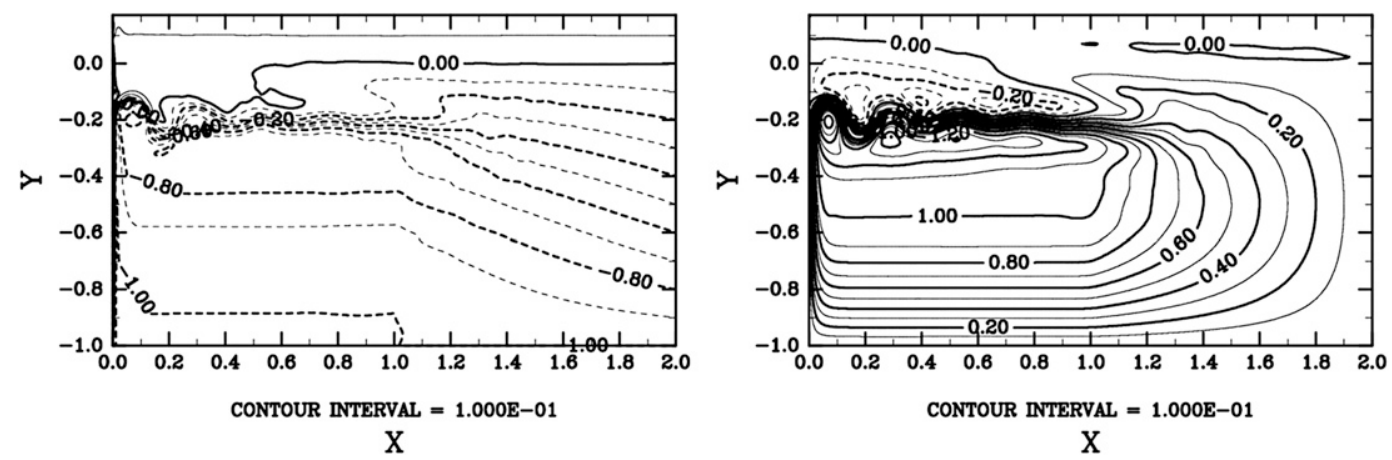

FIG. 5. Result for asymmetric $\hat{w}_{e}$ in the meridional direction about $y=0$. The northern boundary is located at $Y_{N}=0.2$ and $\hat{w}_{e}=0$ for $y>0$ and $\hat{w}_{e}=\sin (\pi y)$ for $-1.0 \leq y \leq 0$. (left) Temporal-mean potential vorticity field divided by $\hat{\beta}$; (right) temporal-mean streamfunction field. Parameters $\delta_{M}=0.30, \delta_{I}=0.9$, and other parameters are as in Fig. 1. A solution of the gyre boundary separation case was given as the initial condition.

separation, the temporal variation of $Y_{\mathrm{Jet}}$ is approximately 0.03, which is larger than the difference in $Y_{\mathrm{Jet}}$ caused by changes in the parameters. Therefore, $Y_{\text {Jet }}$ is regarded as approximately -0.2 in these cases, independent of the parameters. We found that the flow pattern tended to be the premature separated jet case for large $\delta_{M}^{3} / \delta_{I}^{2}$ to which the Reynolds number was proportional and to be the boundary jet case for small $\delta_{M}^{3} / \delta_{I}^{2}$, as expected from Fig. 4a. However, the two solution types coexist in the medium range of $\delta_{M}^{3} / \delta_{I}^{2}$, which suggests the solution type is not determined only by $\delta_{M}^{3} / \delta_{I}^{2}$. Figure $4 \mathrm{~b}$ shows the jet length in the $\delta_{M}-\delta_{I}$ plane. It also has little dependence on parameters for the prematurely separated jet case, although its length tends to increase with increasing $\delta_{I}$. In the gyre boundary jet case, temporal variation in $Y_{\text {Jet }}$ does not occur except for the initial transient; the value remains at $Y_{\mathrm{Jet}}=0.0$, and the jet extends to the eastern boundary in all cases.

Experiments with other initial conditions were also conducted. In the parameter range that showed a gyre boundary jet, similar experiments were conducted with the initial conditions set as the time-mean solution of a premature separation case calculated using slightly different parameters. Except for the cases in which $\delta_{M}=0.2$ and $\delta_{I}=1.06$, the quasi-steady solutions showed prematurely separated jets. The observed dependence on the initial conditions indicates the existence of multiple equilibrium solutions. With the initial conditions set to the solution of the gyre boundary jet case, $Y_{\text {Jet }}$ was 0.0 over the entire parameter range of Fig. 4. Once an extension jet was formed along the free-slip northern boundary, the structure was quite robust and hardly collapsed. For much smaller $\delta_{I}$ of 0.58 and $\delta_{M}=0.3$ (not shown), even if the initial condition was the gyre boundary separation type, the solution eventually evolved to a prematurely separated jet. However, in such a case, because the jet separated from the western boundary declines immediately and did not extend enough, we did not address such weak jet cases in detail below. In symmetric double-gyre cases, the initial gyre boundary jet was also maintained at this position, although the length of the jet decreased. In contrast, as shown in Fig. 5, when the Ekman pumping velocity for $y>0$ was set as a nonsymmetric distribution to $y=0$, a prematurely separated jet was formed and $Y_{\text {Jet }}$ was nearly -0.2 independent of the initial conditions. This suggests that the presence of meridional symmetry in the gyre boundary makes the structure of the gyre boundary jet robust and gives rise to multiple solutions. It is important that $Y_{\text {Jet }}$ is constant at approximately -0.2 for various $\delta_{M}$ and $\delta_{I}$ in the prematurely separated jet case. This case has no intermediate solution with $Y_{\text {Jet }}=-0.10$, for example.

\section{2) DEPENDENCE ON BOUNDARY CONDITION}

In the experiment conducted by Haidvogel et al. (1992), premature separation did not occur under freeslip western boundary conditions; however, when the partial-slip coefficient $\alpha$ increased, so the boundary condition approached the no-slip condition, premature separation was observed. This suggests that the generation of positive vorticity in the viscous boundary layer is important for premature separation to occur (Kiss 2002). As seen in Fig. 1, when the premature separation occurs, high potential vorticity fluid is accumulated in the northern recirculation gyre. This high potential vorticity is generated on the western side of the boundary current, while the low potential vorticity in the southern recirculation gyre is advected from the south. Since the western boundary current is a narrow jet, total relative vorticity integrated over the boundary layer almost vanishes, when the no-slip boundary condition is applied. This could be related to a symmetric structure of northern and southern recirculation gyres. Since the zero total relative vorticity always holds independent of $\delta_{I}$ and $\delta_{M}$, 
it could explain the independence of the latitude of the prematurely separated jet from the parameters. So, we conducted a series of experiments with the partial-slip boundary condition that would tend to give negative total relative vorticity and investigated the latitude of the extension jet on the partial-slip coefficient $\alpha$. In this series of experiments, $\delta_{M}=0.3$ and $\delta_{I}=0.9$; the values of the other parameters were the same as those in the previous experiments.

The dependence of $Y_{\text {Jet }}$ on the partial-slip coefficient is shown in Fig. 6a. As the partial-slip coefficient decreased, the regime changed from the prematurely separated jet case to the gyre boundary jet case. These characteristics are qualitatively consistent with that in Haidvogel et al. (1992), although the value of $\alpha$ where the regime transition occurs is larger than that in Haidvogel et al. (1992) (while the value is from 3 to 6.5 in their experiment, it is estimated to be about 48 to 61 in the present study using their scaling). However, we found that the regime also depends on the initial conditions, so a single critical value of $\alpha$ cannot be defined. Haidvogel et al. (1992) also saw a gradual transition but ours was abrupt. In the experiments of Haidvogel et al. (1992), broad oscillation of the western boundary current separation point was observed at relatively small $\alpha$; however, such broad oscillation did not occur in our experiments. These discrepancies would come from the differences in the viscosity term for which they used the biharmonic form and/or existence of a slip northern boundary. The value of $Y_{\mathrm{Jet}}$ is nearly -0.2 independent of $\alpha$ and total positive vorticity (see also Fig. 6b) when the premature separation occurs. When the boundary jet occurs the values of the total positive vorticity were much larger because the upper-layer circulation is significantly enhanced. This result supports the idea that $Y_{\text {Jet }}$ does not depend on the detailed structure of the western boundary region in a prematurely separated jet case.

\section{3) OTHER PARAMETERS}

As mentioned in section 2, the ratio of the thickness of the upper and lower layers $\left(H_{1} / H_{2}\right)$ was also a parameter that determined the circulation pattern in this model. The ratio of $H_{1}$ to $H_{2}$ is closely related to baroclinic instability. When the upper-layer eastward velocity is $U_{1}$ and the lower layer is at rest, the criterion for the occurrence of baroclinic instability is $U_{1}>\hat{\beta} / F_{2}=2\left(1+H_{2} / H_{1}\right) \delta_{I}^{-2}$. As $H_{1} / H_{2}$ increases, the instability becomes stronger. Because the baroclinic instability tends to mix the potential vorticity in the lower layer, the meridional width of the potential vorticity homogenized region in the lower layer tends to be narrower and barotropic recirculation gyres are weakened as $H_{1} / H_{2}$ decreases.

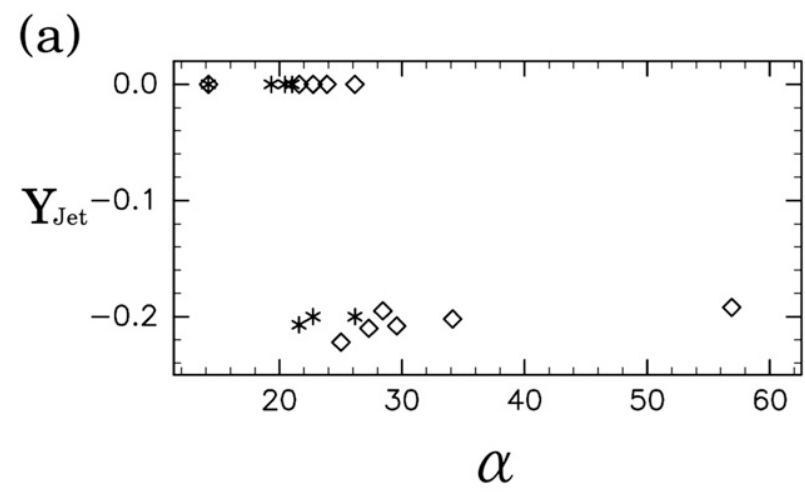

(b)

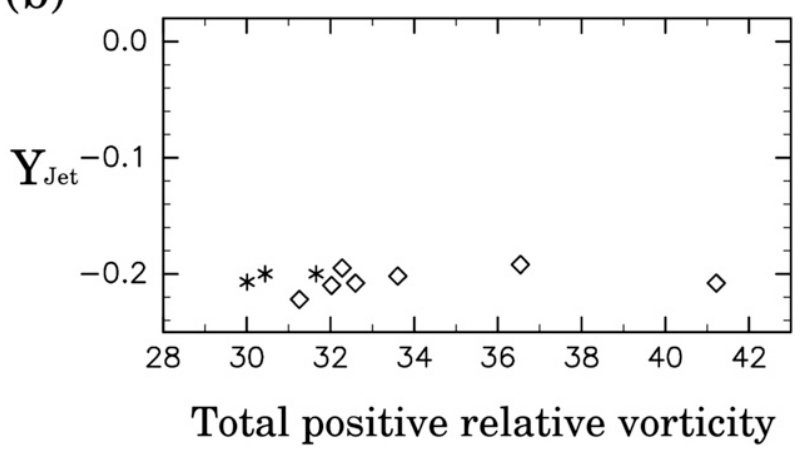

FIG. 6. The $y$ coordinate of the extension jet $Y_{\text {Jet }}$. (a) As a function of the partial-slip coefficient $\alpha$ and (b) as a function of total positive relative vorticity in the western boundary layer, which is the maximum meridional velocity minus the velocity at the western boundary along $y=-0.5$. Symbols denote the data points, where $\diamond$ denotes that experiments were run from the rest and $*$ denotes experiments were run from the mean solution with prematurely separated jet under the no-slip western boundary condition. In (b), the plots are made only for the prematurely separated jet case, and the total positive vorticity is much larger for the gyre boundary jet case.

Figure 7 shows the results of experiments performed under four different $H_{1} / H_{2}$ with the no-slip western boundary condition and $\left(\delta_{M}, \delta_{I}\right)=(0.3,0.9)$, that is, the same values used in the previous section. Here, we changed $H_{1} / H_{2}$ from $1 / 1$ to $1 / 16$. If we assume that the other dimensional parameters including $R_{d}$ are fixed as in Table $1, H_{1}$ and $H_{2}(\mathrm{~m})$ used for the experiments in Fig. 7 correspond to $\left(H_{1}, H_{2}\right) \approx(1600,1600),(1200,2400)$, $(900,7200)$, and $(850,13600)$. Smaller $H_{1} / H_{2}$ was associated with weaker flow in the lower layer. In the case with $H_{1} / H_{2}=1 / 8$, there was still a closed contour under the southern recirculation; the pattern of the $\psi_{2}$ contours is similar to the case with $H_{1} / H_{2}=1 / 4$ (Fig. 1d). When $H_{1} / H_{2}=1 / 16$ the western boundary current did not separate until it reached the northern boundary. In this case, the lower layer was almost at rest. This suggests that an active lower layer is important for premature separation to occur in the rectangular basin, although the reason is 

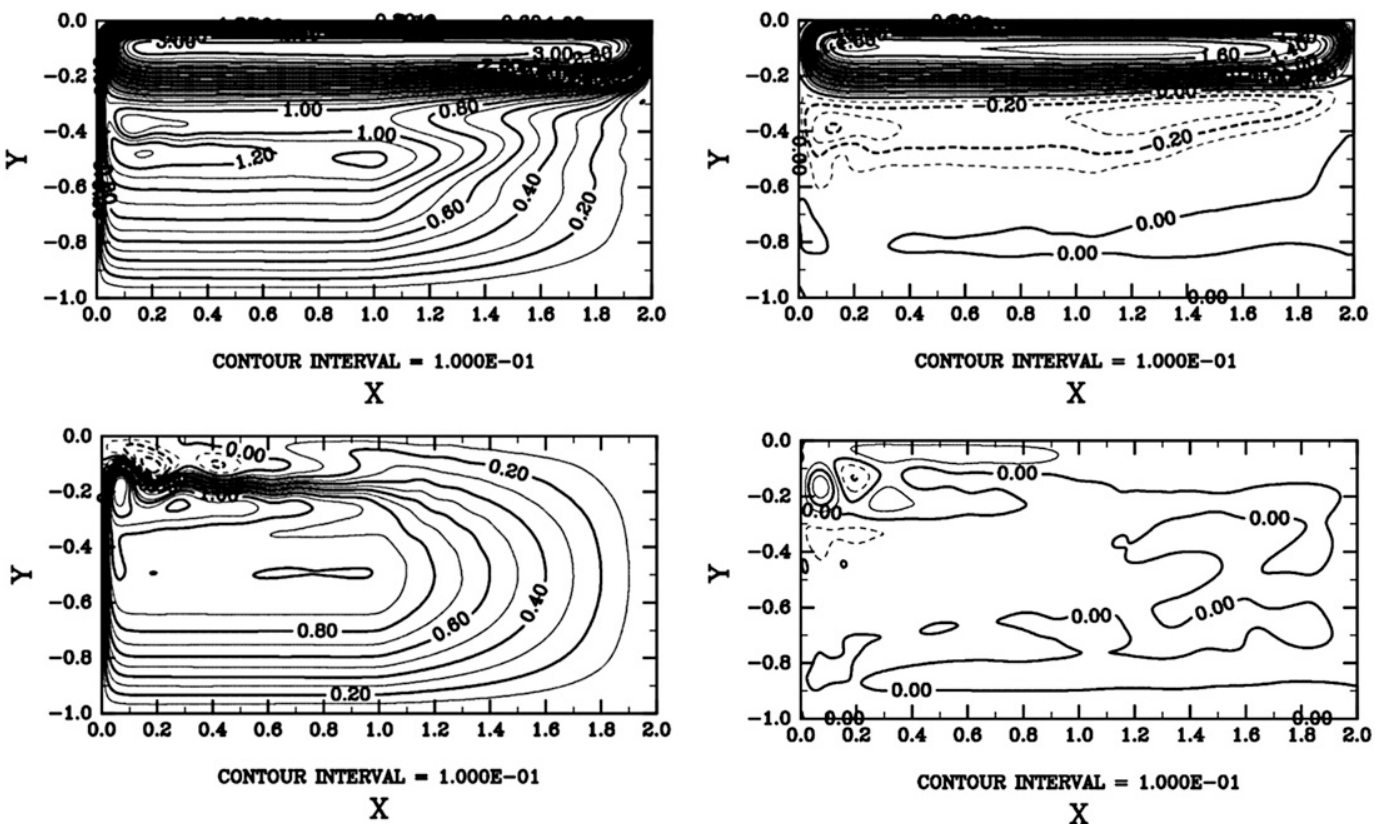

$\mathrm{X}$
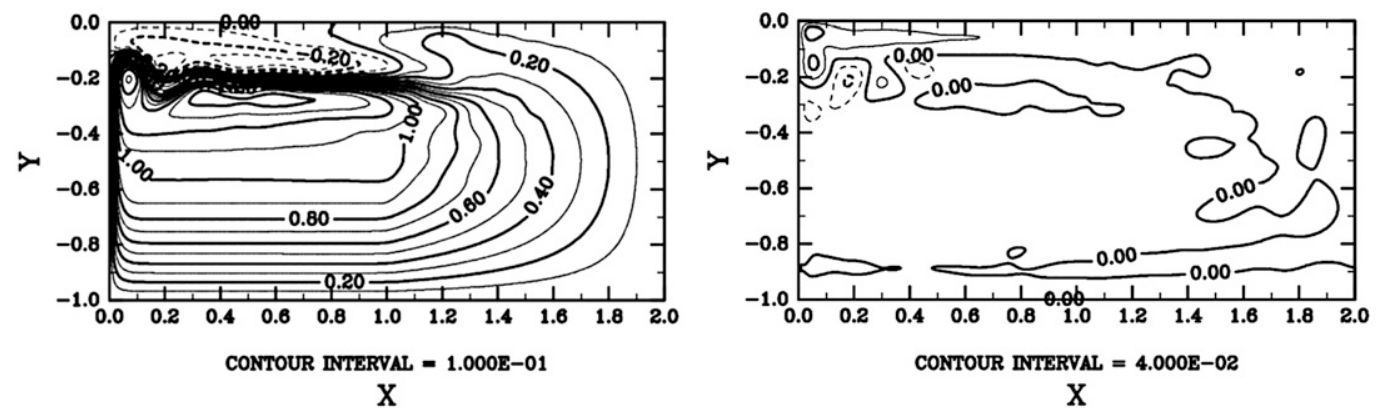

$\mathrm{X}$
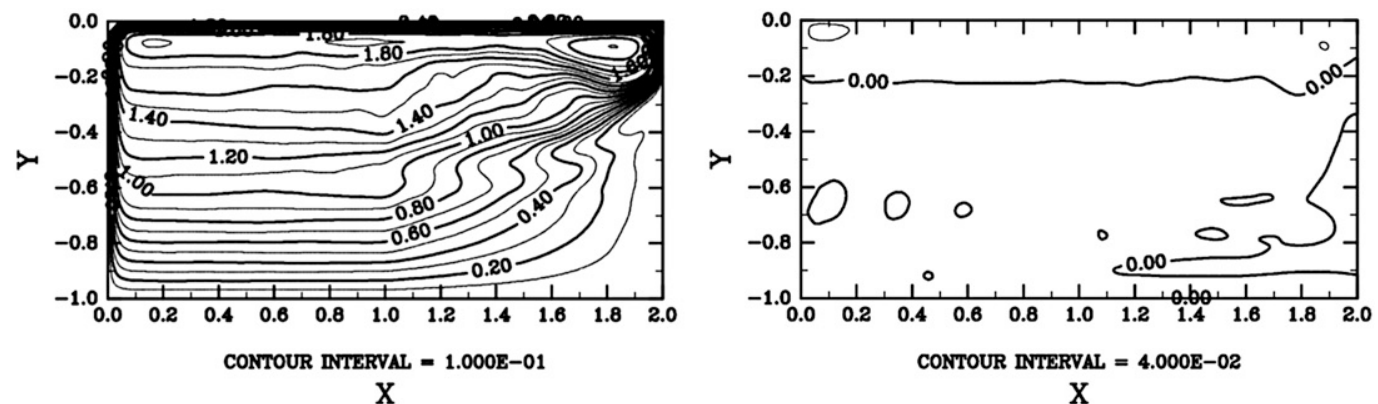

FIG. 7. Time-mean streamfunction field for each layer thickness ratio. (top to bottom) $H_{1} / H_{2}=1 / 1, H_{1} / H_{2}=1 / 2$, $H_{1} / H_{2}=1 / 8, H_{1} / H_{2}=1 / 16$; other parameters are as in Fig. 1. (left) Upper layer; (right) lower layer. The contour interval is 0.04 for the last two panels on the right side and 0.1 for the others.

still not clear. This is consistent with the experiments by Pierini (2006), who used a 1.5-layer model, in which the latitude of the extension jet seems realistic; however, relative to the Sverdrup circulation, the jet was located along the gyre boundary. To confirm the necessity of two active layers, we carried out experiments using a onelayer barotropic model and a 1.5-layer model. The quasisteady solutions of these models showed the gyre boundary jet case, even when we gave the streamfunction in the prematurely separated jet case as their initial conditions (not shown).

In the cases of $H_{1} / H_{2}=1 / 2$ and $1 / 8$, premature separation occurred as in the standard case. In these cases, $Y_{\text {Jet }}$ was also nearly -0.2 . This suggests that $Y_{\text {Jet }}$ is also almost independent of $H_{1} / H_{2}$ once premature separation occurs. For $H_{1} / H_{2}=1$, the flow pattern was of the gyre boundary jet type. However, the anticyclonic recirculation gyre near the northern boundary occupied 

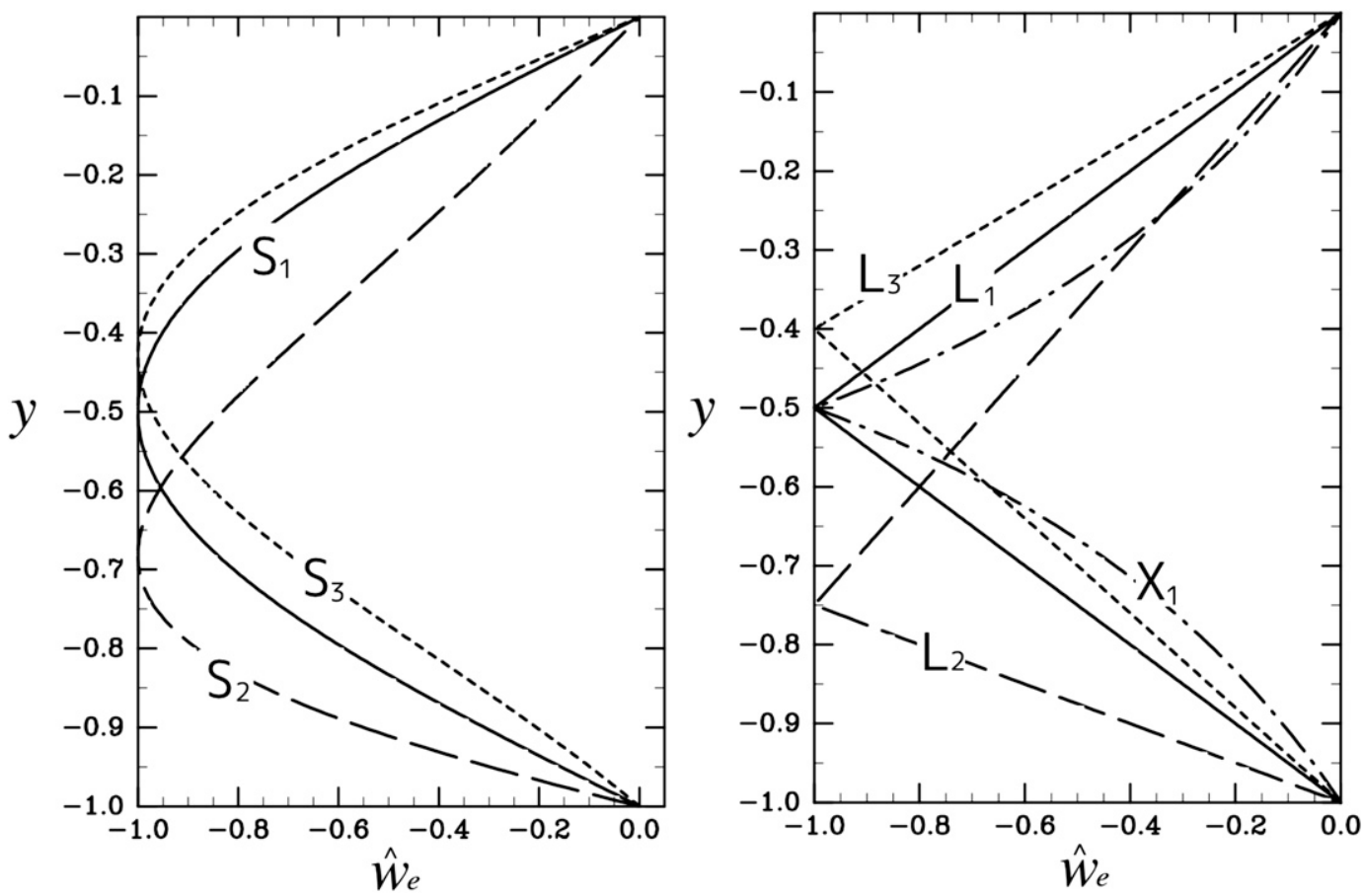

FIG. 8. The meridional distribution of the Ekman pumping velocity $\hat{w}_{e}$. The horizontal axis represents the Ekman pumping velocity. For definitions of the labels, see Table 3.

more than one-fourth of the entire basin and formed a much stronger circulation than in the previous experiments. The vigorous horizontal mixing caused by the baroclinic instability produced a wide region of homogenized potential vorticity in the lower layer, and this homogenized region generated the barotropic anticyclonic gyre along the boundary. In the double-gyre case, while the gyre boundary jet occurred, strong circulation of this type was not formed because of the potential vorticity mixing through the subpolar/subtropical intergyre boundary.

In addition to the basic value of $\hat{r}=10^{-3}$, dependence on $\hat{r}$ was also investigated for the values of 0 and $10^{-4}$ under $\delta_{M}=0.3, \delta_{I}=0.9$ and $H_{1} / H_{2}=1 / 4$. All values of $\hat{r}$ produced premature separation and $Y_{\mathrm{Jet}}$ was also -0.2 , although many more eddies were observed for smaller $\hat{r}$.

\section{c. Dependence on meridional distribution of Ekman pumping velocity}

As shown in the previous subsections, the latitude of the eastward jet hardly depends on the parameters once premature separation occurs. In addition, Nakano et al. (2008) showed that the latitude of the extension jet does not depend on the shape of the western boundary. These results lead us to expect that the factor that determines the latitude of the extension jet is the distribution of external forcing. In all previous experiments, we used the meridional distribution of the Ekman pumping velocity of $\hat{w}_{e}=\sin \pi y$. Therefore, we investigated the dependence of the latitude of the extension jet on the distribution of $\hat{w}_{e}$ with $\left(\delta_{M}, \delta_{I}\right)=(0.3,0.9), H_{1} / H_{2}=1 / 4$, $\hat{r}=10^{-3}$, and the no-slip western boundary condition. The distributions of Ekman pumping velocity that were investigated are summarized in Fig. 8 and Table $3 ; S_{k}$ are sinusoidal, $L_{k}$ are triangular ( $k=1$ to 3 ), and $X_{1}$ is of cusp shape. In the $L_{2}$ case, $\delta_{I}$ was set to 1.1 because the strength of the jet was weakened by widening the eastward Sverdrup flow region in the meridional direction, and the jet length was short relative to the other cases under the same value of $\delta_{I}$.

As an example of the results, Fig. 9 shows the meridional distribution of the zonal-mean potential vorticity and streamfunction for $L_{1}, X_{1}$, and $L_{2}$. The values of $Q_{1}(y)$ and $\Psi_{1}(y)$ are consistent with the values estimated by the Sverdrup balance except for near the jet region similar to the $S_{1}$ case (see Fig. 3b), although they are slightly different near the center of the gyre where $\partial \hat{\psi}_{\mathrm{Sv}} / \partial y$ is discontinuous but $\psi_{1}$ is smooth. In contrast to the results of previous subsections, the latitude of the jet was significantly different for each $\hat{w}_{e}$, as shown in Fig. 10a and Table 3. The figure indicates two trends in $Y_{\text {Jet }}$. The first is that $Y_{\text {Jet }}$ tends to shift in the direction in which the center of the Sverdrup circulation corresponding to the latitude of maximum $\left|\hat{w}_{e}\right|, Y_{C}$, is shifted. The second is that $Y_{\text {Jet }}$ depends on the curvature of the meridional distribution of $\hat{w}_{e}$; a larger curvature is 
TABLE 3. Meridional distributions of given Ekman pumping velocities with summarized results.

\begin{tabular}{|c|c|c|c|c|c|}
\hline & Meridional distribution of $\hat{w}_{e}(y)$ & $Y_{C}$ & $\left.y\right|_{\psi_{\mathrm{Sv}}=0.5}$ & $Y_{\text {Jet }}$ & $Y_{\mathrm{Sv}}\left(\Psi_{\mathrm{Jet}}\right)$ \\
\hline$S_{1}$ & $\hat{w}_{e}(y)=\sin \pi y$ & -0.5 & -0.167 & -0.207 & -0.183 \\
\hline$S_{2}$ & $\hat{w}_{e}(y)=\sin \pi\left[(1-a) y+a y^{3}\right] \quad a=0.5$ & -0.68 & -0.305 & -0.282 & -0.277 \\
\hline$S_{3}$ & $\hat{w}_{e}(y)=\sin \pi\left[(1-a) y+a y^{3}\right] \quad a=-0.2$ & -0.43 & -0.140 & -0.176 & -0.149 \\
\hline$L_{1}$ & $\hat{w}_{e}(y)= \begin{cases}2 y & (y \geq-0.5) \\
-2(y+1) & (y<-0.5)\end{cases}$ & -0.5 & -0.250 & -0.231 & -0.225 \\
\hline$L_{2}$ & $\hat{w}_{e}(y)= \begin{cases}\frac{4 y}{3} & (y \geq-0.75) \\
-4(y+1) & (y<-0.75)\end{cases}$ & -0.75 & -0.375 & -0.306 & -0.302 \\
\hline$L_{3}$ & $\hat{w}_{e}(y)= \begin{cases}\frac{5 y}{2} & (y \geq-0.4) \\
-\frac{5(y+1)}{3} & (y<-0.4)\end{cases}$ & -0.4 & -0.200 & -0.193 & -0.183 \\
\hline$X_{1}$ & $\hat{w}_{e}(y)= \begin{cases}\frac{y}{(1+y)} & (y \geq-0.5) \\
\frac{(1+y)}{y} & (y<-0.5)\end{cases}$ & -0.5 & -0.333 & -0.293 & -0.293 \\
\hline
\end{tabular}

associated with smaller $\left|Y_{\text {Jet }}\right|$, which could be confirmed by comparing the $S_{1}, L_{1}$ and $X_{1}$ cases. Because of Eq. (6), with the same $Y_{C}, Y_{\mathrm{Jet}}$ in the case with positive (negative) curvature of $\hat{\psi}_{\mathrm{Sv}}, d^{2} \hat{\psi}_{\mathrm{Sv}} / d y^{2}$, occurs at a higher (lower) latitude than in the case with zero curvature of $\hat{\psi}_{\mathrm{Sv}}$.

Because the extension jet connected to the Sverdrup interior region smoothly and the contours of all streamfunction values concentrate in the jet region, it was expected that the streamfunction of the center of the jet, $\Psi_{\mathrm{Jet}}\left[=\Psi_{1}\left(Y_{\mathrm{Jet}}\right)\right]$, would be close to the average of the minimum and maximum $\hat{\psi}_{\mathrm{Sv}}$ values, which was 0.5 in our experiments. Figure $10 \mathrm{~b}$ shows the relation between $Y_{\mathrm{Jet}}$ and the $y$ coordinate where $\hat{\psi}_{\mathrm{Sv}}=0.5$ [hereinafter $\left.Y_{\mathrm{Sv}}(0.5)\right]$. The correlation between $Y_{\mathrm{Jet}}$ and $Y_{\mathrm{Sv}}(0.5)$ is better than that between $Y_{\text {Jet }}$ and $Y_{C}$. When the curvature of $\hat{\psi}_{\mathrm{Sv}}$ is negative [i.e., $\left(d^{2} \hat{\psi}_{\mathrm{Sv}} / d y^{2}\right)<0$ ] as the $S_{1}$ case, $Y_{\mathrm{Sv}}(0.5)$ shifts northward and vice versa, although $\Psi_{\mathrm{Jet}}$ varies between 0.4 and 0.6 (see Fig. 11a). The difference of $\Psi_{\text {Jet }}$ from 0.5 probably comes from the asymmetry of the jet and reduction of the $\Psi_{1}\left(Y_{C}\right)$ value for a smooth connection in the case with discontinuous $\left.\left(\partial \hat{\psi}_{\mathrm{Sv}} / \partial y\right)\right|_{y=Y_{C}}$ as seen in Fig. 9.

The above discussion roughly interprets the dependency of $Y_{\text {Jet }}$ on the meridional profile of $\hat{w}_{e}$, which suggests that $Y_{\text {Jet }}$ would be equal to the $y$ coordinate where $\hat{\psi}_{\mathrm{Sv}}(y)=\Psi_{\text {Jet }}$ and $Y_{\mathrm{Sv}}\left(\Psi_{\mathrm{Jet}}\right)$. However, Fig. 11b shows that the difference between $Y_{\mathrm{Jet}}$ and $Y_{\mathrm{Sv}}\left(\Psi_{\mathrm{Jet}}\right)$ is large in $S_{1}$ and $S_{3}$ cases, as compared with that in cases $S_{2}, L_{k}$, and $X_{1}$. As the curvature in $S_{2}$ is negative but its magnitude is much smaller than that in $S_{1}$ around $y=Y_{\text {Jet }}$, Fig. $11 \mathrm{~b}$ suggests that negative curvature in $\hat{\psi}_{\mathrm{Sv}}$ tends to shift the jet southward. To illustrate this, an integral condition given in the western part of the basin,

$$
\int_{Y_{S}}^{Y_{N}} \bar{\psi}_{1} d y \approx \int_{Y_{S}}^{Y_{N}} \hat{\psi}_{\mathrm{Sv}} d y,
$$

was considered, where the overbar denotes the time mean. This condition is derived from Eqs. (1) and (2) under several assumptions when premature separation occurs. The derivation is shown in the appendix. Figure 12 is the plot of the meridional integral of $\hat{\psi}_{\mathrm{Sv}}$ and that of the time- and spatial-averaged $\psi_{1}$ and shows that Eq. (7) is substantially satisfied in all cases.

Figure 13 is the schematic to interpret the relation of $Y_{\text {Jet }}$ and Eq. (7) above. Equation (7) states that the area $\mathrm{A}$ is equal to the area B in Fig. 13. When $\left(d^{2} \hat{\psi}_{\mathrm{Sv}} / d y^{2}\right)=0$, $Y_{\text {Jet }}$ coincides with $Y_{\mathrm{Sv}}\left(\Psi_{\text {Jet }}\right)$ as in the left panel. On the other hand, when $\left(d^{2} \hat{\psi}_{\mathrm{Sv}} / d y^{2}\right)<0$ as in $\hat{w}_{e}=\sin \pi y, Y_{\mathrm{Jet}}$ must be smaller than $Y_{\mathrm{Sv}}\left(\Psi_{\mathrm{Jet}}\right)$ if $\Psi_{\mathrm{Jet}}$ is the mean of the local maximum and minimum of $\Psi_{1}(y)$ on either side of the jet. (We confirmed that the midpoint between the local maximum and minimum of $\Psi_{1}$ coincided with $Y_{\text {Jet }}$ within the width of one grid cell in our experimental results.) This explains the relation between $Y_{\text {Jet }}$ and $Y_{\mathrm{Sv}}\left(\Psi_{\mathrm{Jet}}\right)$ for $S_{1}$ and $S_{3}$ cases. Similarly, this explanation makes us expect that $Y_{\mathrm{Jet}}=Y_{\mathrm{Sv}}\left(\Psi_{\mathrm{Jet}}\right)$ for $L_{k}$ cases and $Y_{\mathrm{Jet}}>Y_{\mathrm{Sv}}\left(\Psi_{\mathrm{Jet}}\right)$ for $X_{1}$ case, but in our experiments, $Y_{\mathrm{Jet}}$ was slightly smaller than $Y_{\mathrm{Sv}}\left(\Psi_{\mathrm{Jet}}\right)$ for $L_{k}$ cases and $Y_{\mathrm{Jet}}$ was almost equal to $Y_{\mathrm{Sv}}\left(\Psi_{\mathrm{Jet}}\right)$ for the $X_{1}$ case. This discrepancy perhaps comes from the distortion of the upper-layer streamfunction field caused by the discontinuity in $\partial \psi_{\mathrm{Sv}} / \partial y$ at $y=Y_{C}$ as seen in Fig. 9.

Therefore, we can conclude that $\Psi_{\text {Jet }}$ tends to take a value around the mean between the maximum and minimum of $\hat{\psi}_{\mathrm{Sv}}$, and the jet axis, $Y_{\mathrm{Jet}}$, is located slightly 

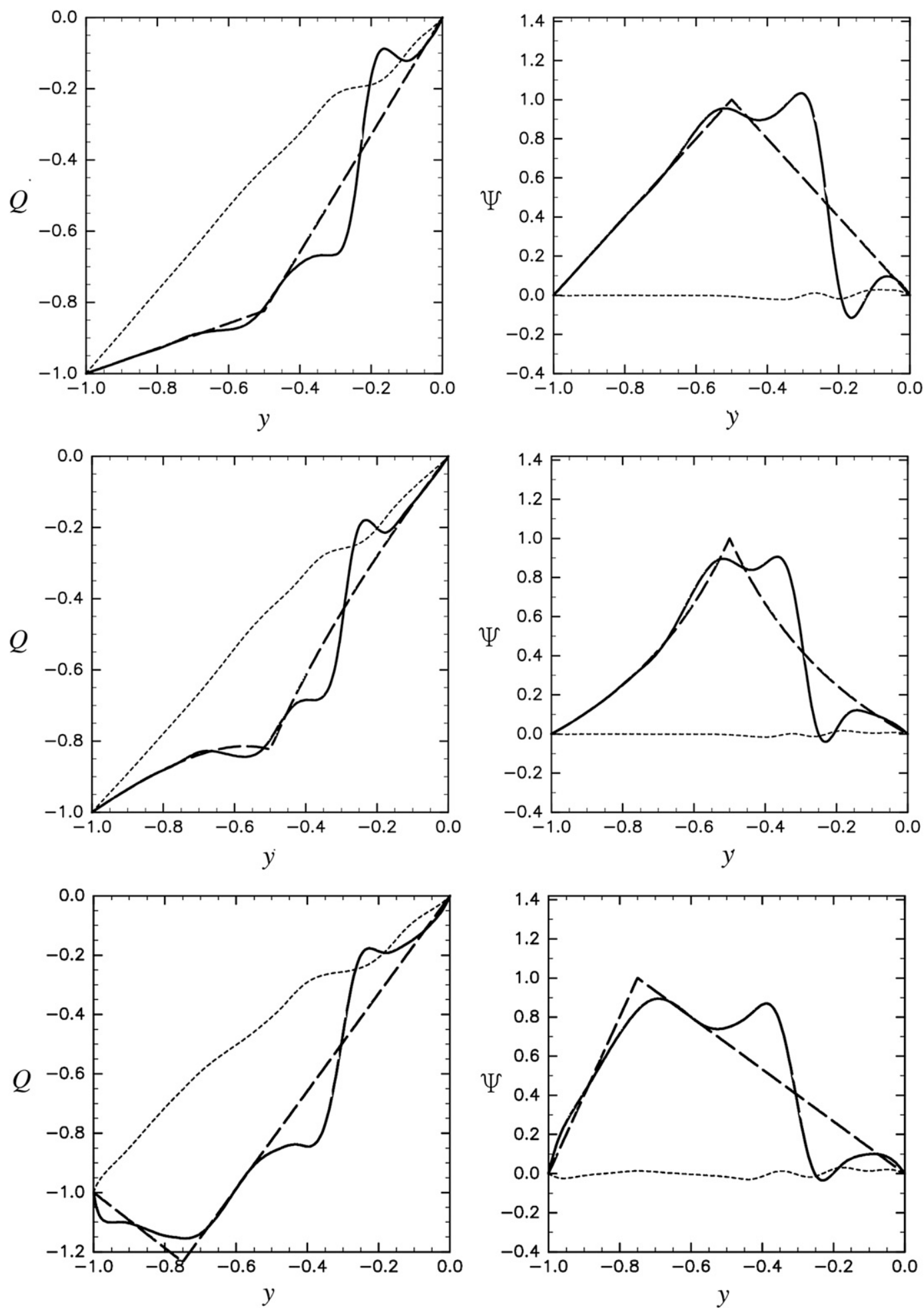

FIG. 9. Meridional distribution of the temporal-mean potential vorticity and streamfunction; definitions are the same as in Fig. 3: (top) $L_{1}$; (middle) $X_{1}$; and (bottom) $L_{2}$. (left) Potential vorticity and (right) streamfunction in the upper layer (solid line) and the lower layer (dotted line) averaged in the zonal direction from $x=0.5$ to $x=1.0$. The dashed lines are $\hat{q}_{\mathrm{Sv}}$ and $\hat{\psi}_{\mathrm{Sv}}$, which were estimated from the Sverdrup balance for the upper layer. 
(a)

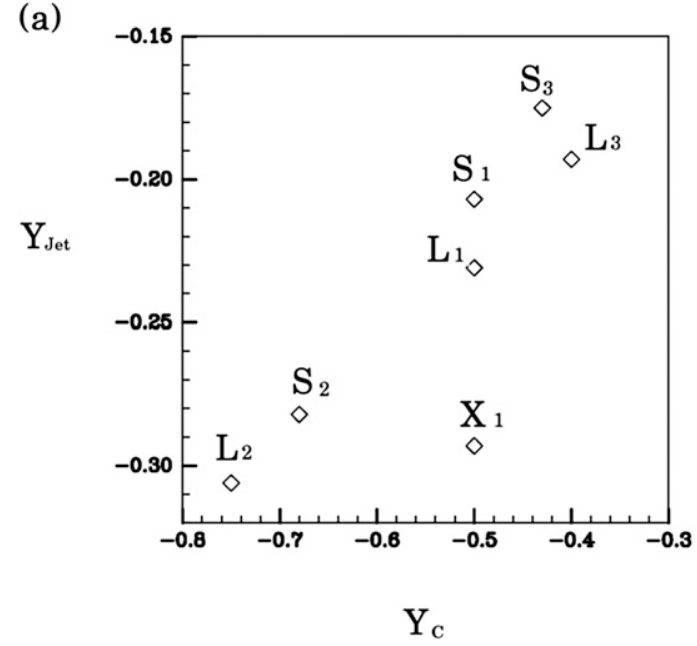

(b)

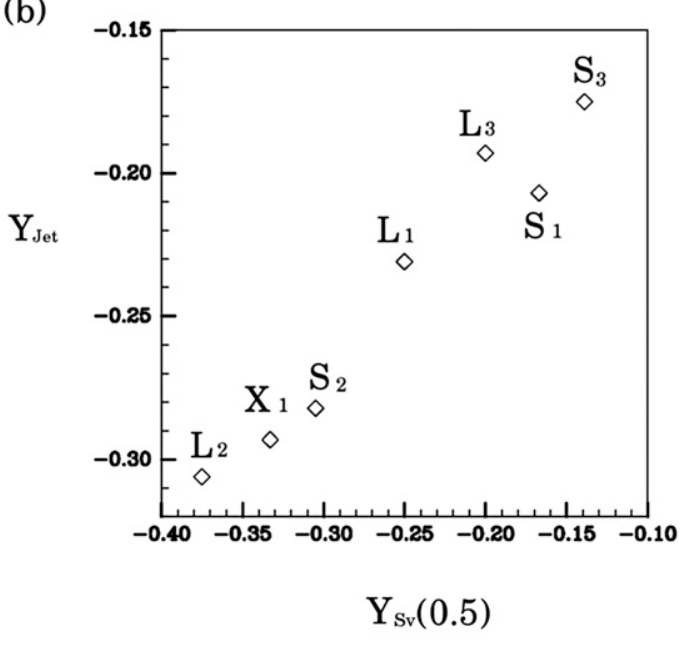

FIG. 10. (a) The $y$ coordinate of the extension jet $Y_{\text {Jet }}$ as a function of the $y$ coordinate of the center of the Sverdrup circulation $Y_{C}$. (b) Jet axis $Y_{\mathrm{Jet}}$ as a function of the $y$ coordinate at which $\hat{\psi}_{\mathrm{Sv}}=0.5$ and $Y_{\mathrm{Sv}}(0.5)$. The labels in the figure correspond to Table 3.

to the south of the latitude expected from $\hat{\psi}_{\mathrm{Sv}}(y)=\Psi_{\mathrm{Jet}}$ for the case of $\hat{w}_{e}=\sin \pi y$.

\section{Discussion and conclusions}

The parameter dependence of the latitude of the eastward extension jet that separates from the western boundary was investigated using a two-layer quasigeostrophic model in a rectangular basin. The investigated parameters included the inertial boundary layer width $\delta_{I}$, the viscous boundary layer width $\delta_{M}$, the partial-slip coefficient $\alpha$, the ratio of the upper- and lower-layer thickness $H_{1} / H_{2}$, and the coefficient of bottom friction $\hat{r}$.
It was shown that the patterns of circulation that included the extension jet could be classified into two patterns. One was the prematurely separated jet case, and the other was the gyre boundary jet case. When $\delta_{I}$ was smaller and $\delta_{M}$ was larger than certain values, the solution tended to show the premature separation pattern where the change occurred abruptly, and there was no intermediate solution. Meanwhile, in the parameter range investigated, almost everywhere there are multiple solutions depending on initial conditions. Such character is common in many previous studies on the nonlinear ocean circulation (e.g., Jiang et al. 1995; Cessi and Ierley 1995; McCalpin and Haidvogel 1996; Primeau (a)

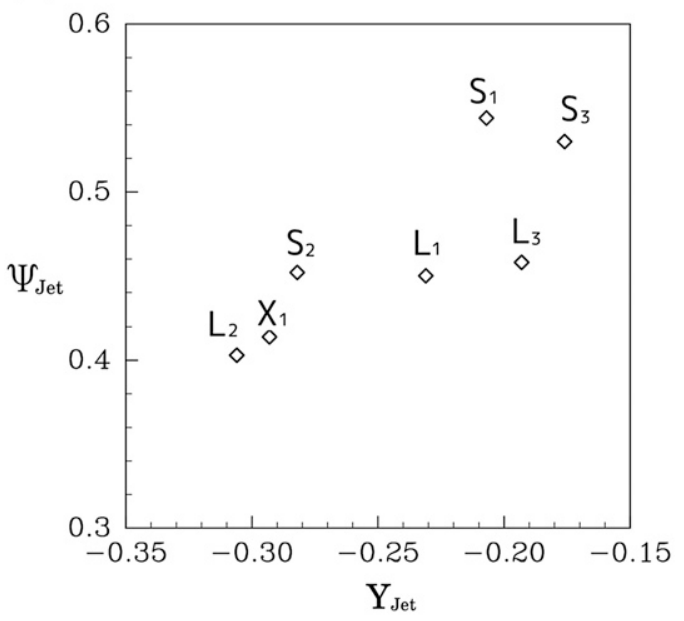

(b)

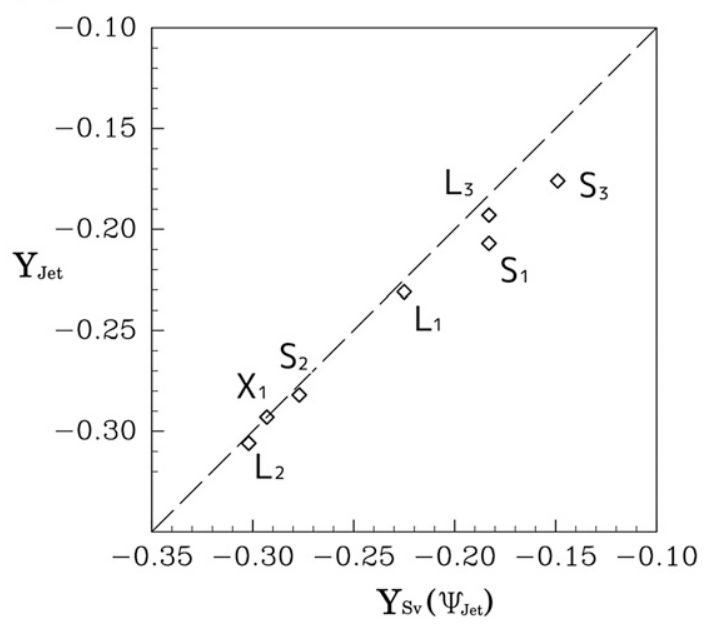

FIG. 11. (a) The streamfunction value at the axis of the jet $\Psi_{\text {Jet }}$ as a function of the $y$ coordinate of the extension jet $Y_{\mathrm{Jet}}$. (b) Jet axis $Y_{\mathrm{Jet}}$ as a function of the $y$ coordinate at which $\hat{\psi}_{\mathrm{Sv}}=\Psi_{\mathrm{Jet}}$ and $Y_{\mathrm{Sv}}\left(\Psi_{\mathrm{Jet}}\right)$. The dashed line shows $Y_{\mathrm{Jet}}=Y_{\mathrm{Sv}}\left(\Psi_{\mathrm{Jet}}\right)$ 


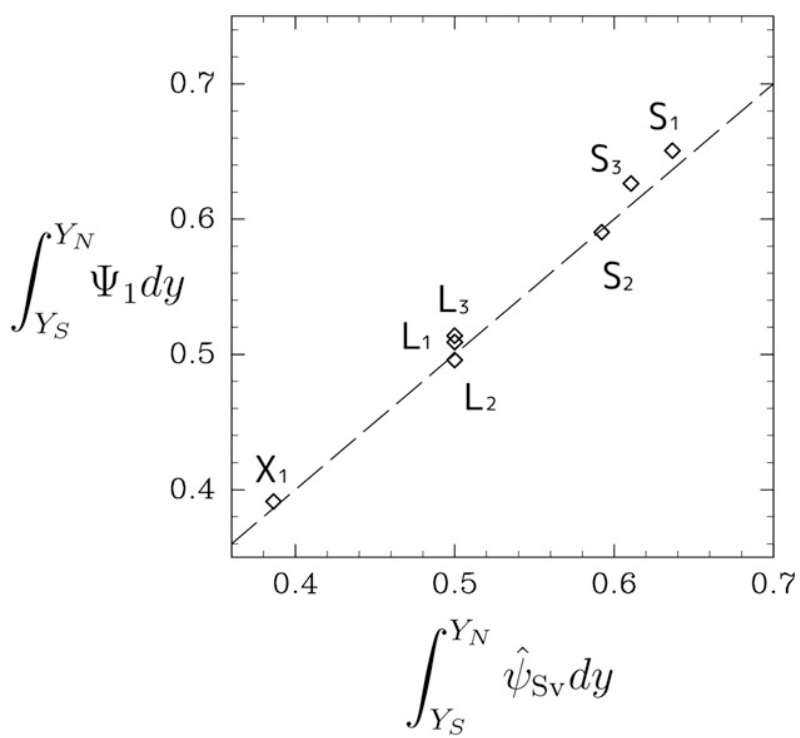

FIG. 12. A plot of the meridionally integrated value of time-mean streamfunction against that of the Sverdrup streamfunction at arbitrary longitude in the west-half region. The dashed line shows $\int \psi_{\mathrm{Sv}} d y=\int \Psi_{1} d y$

and Newman 2007). Our result is different from the result obtained using the 1.5-layer model driven by double-gyre wind forcing (Jiang et al. 1995) that showed continuous change of separation latitude with the strength of the forcing as well as from the result obtained using a barotropic model (Cessi and Ierley 1995). Their variation of the separation point is caused by an asymmetric structure with a confluent point of southward and northward western boundary currents. In our 1.5-layer model and barotropic model experiments, the prematurely separated jet could not be seen even when the initial condition was of the prematurely separated jet case.

For the prematurely separated jet case, $\delta_{M}, \delta_{I}, H_{1} / H_{2}$, and $\hat{r}$ had little impact on $Y_{\text {Jet }}$. In addition, $Y_{\text {Jet }}$ did not depend on the western boundary condition in this case. These findings suggest that when premature separation occurs, $Y_{\mathrm{Jet}}$ is independent of the detailed configuration of the western boundary. In contrast, it was found that $Y_{\text {Jet }}$ was strongly affected by the meridional distribution of the Ekman pumping velocity $w_{e}$.

We especially studied the effects on $Y_{\text {Jet }}$ of the location at the center of the Sverdrup circulation and the curvature of the Sverdrup streamfunction in the western $\operatorname{basin}\left(\hat{\psi}_{\mathrm{Sv}}\right)$. It was found that the streamfunction value at the jet $\Psi_{\text {Jet }}$ tends to take a value around half of the streamfunction at the center of the Sverdrup circulation, and the jet axis $Y_{\text {Jet }}$ is located slightly to the south of the latitude expected from $\hat{\psi}_{\mathrm{Sv}}(y)=\Psi_{\mathrm{Jet}}$ for the case of $\hat{w}_{e}=\sin \pi y$. In the real ocean, the central latitude of the Sverdrup circulation for the subtropical region is approximately $30^{\circ} \mathrm{N}$, the gyre boundary of the subtropical and subpolar gyre is approximately $46^{\circ} \mathrm{N}$ as estimated by zonalmean wind stress curl (Milliff et al. 2004), the latitude of the Kuroshio Extension is approximately $34^{\circ} \mathrm{N}$, and the median latitude of the maximum Sverdrup streamfunction is $35^{\circ} \mathrm{N}$ (Nakano et al. 2008). Our present results are qualitatively consistent with these characteristics.

Meanwhile the integral condition of Eq. (7) is satisfied in all our experiments. From Eq. (7), the relationship for potential vorticity is directly derived as

$$
\int_{Y_{S}}^{Y_{N}} \bar{q}_{1} d y \approx \int_{Y_{S}}^{Y_{N}} \hat{q}_{\mathrm{Sv}} d y
$$

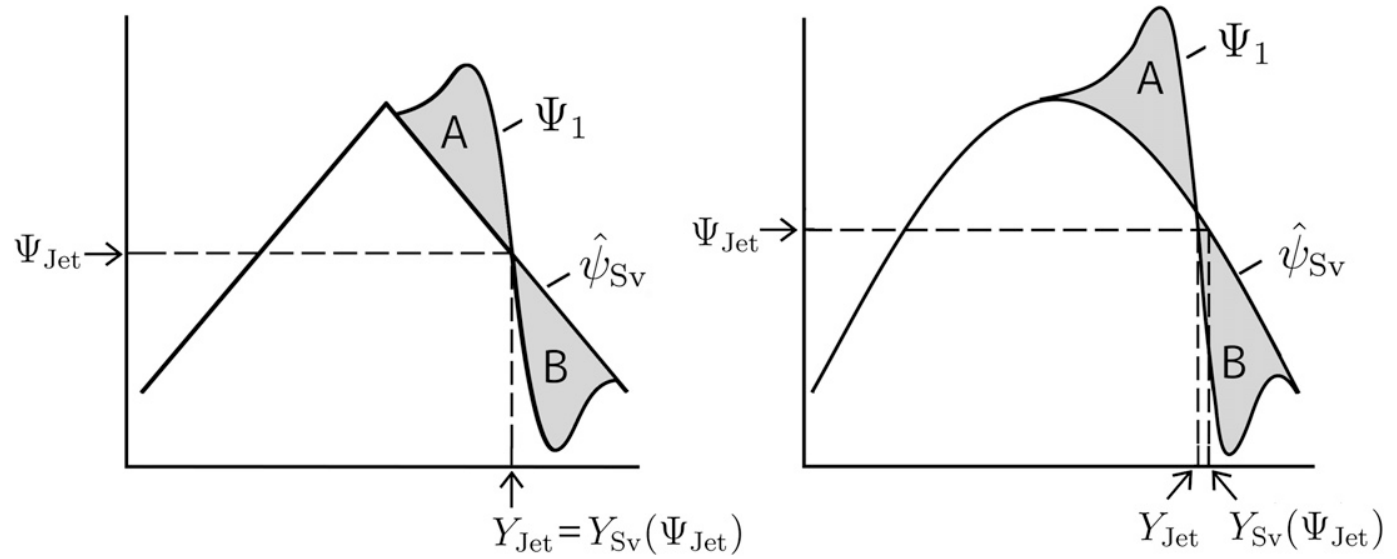

FIG. 13. Schematic illustration of the change in meridional direction of $Y_{\text {Jet }}$ arising from the change in $\hat{\psi}_{\mathrm{SV}}$ distribution. The area of the shadow denoted by A is equal to that by B when Eq. (7) is maintained. Because $\Psi_{\text {Jet }}$ takes the middle value of the local maximum value and local minimum value of $\Psi_{1}$, (left) $Y_{\mathrm{Sv}}\left(\Psi_{\mathrm{Jet}}\right)$ should correspond to $Y_{\mathrm{Jet}}$ in the case of $\partial^{2} \hat{\psi}_{\mathrm{Sv}} / \partial y^{2}=0$, and (right) $Y_{\mathrm{Sv}}\left(\Psi_{\mathrm{Jet}}\right)$ should be to the north from $Y_{\mathrm{Jet}}$ in the case of $\partial^{2} \hat{\psi}_{\mathrm{Sv}} / \partial y^{2}<0$. 
which means that $\hat{q}_{\mathrm{Sv}}$ was redistributed to the meridional distribution of the potential vorticity $q_{1}$ in the region involving the extension jet or recirculations. This supports the above conclusion that $Y_{\text {Jet }}$ is more influenced by the interior Sverdrup region than by the western boundary layer region.

In the present study, we did not provide a forcing in the western half of the region. However, because wind above a real ocean blows across the entire basin, the extension jet would be affected by wind forcing, which caused the reduction of the jet length in our experiments (see Figs. 1 and 5). Because the jet connects to the Sverdrup region at the tip of the jet, it is necessary to understand the effect of the southward Sverdrup flow on the jet length and jet latitude more accurately.

Acknowledgments. The authors thank two anonymous reviewers for their helpful comments. This study was supported by Grant-in-Aids for Scientific Research of the Ministry of Education, Culture, Sports, Science and Technology, Japan (21540447 and 22106006). GFDDENNOU Library was used for drawing figures. Numerical calculations were partly performed on the HITAC SR16000, Hokkaido University.

\section{APPENDIX}

\section{Integral Condition for Streamfunction in the Western Region}

The derivation of Eq. (7) follows from potential vorticity equations, Eq. (1) in the upper layer, integrated from $Y_{S}$ to $Y_{N}$ in the meridional direction and from $X_{E}$ to an arbitrary longitude in the western half region, $x$, in the zonal direction outside the western boundary current region. The area integration of Eq. (1) yields

$$
\begin{aligned}
& \int_{Y_{S}}^{Y_{N}} \int_{X_{E}}^{x}\left\{\frac{\partial q_{1}}{\partial t}+\frac{1}{\hat{\beta}}\left[\frac{\partial}{\partial x}\left(-\frac{\partial \psi_{1}}{\partial y} q_{1}\right)+\frac{\partial}{\partial y}\left(\frac{\partial \psi_{1}}{\partial x} q_{1}\right)\right]\right\} d x d y \\
& \quad=\int_{Y_{S}}^{Y_{N}} \int_{X_{E}}^{x} w_{e} d x d y
\end{aligned}
$$

where we assumed that the viscous terms are sufficiently small and are neglected. With boundary conditions of no-normal flow, Eq. (A1) reduces to

$$
\begin{aligned}
& \frac{\partial}{\partial t} \int_{Y_{S}}^{Y_{N}} \int_{X_{E}}^{x} q_{1} d x d y-\left.\frac{1}{\hat{\beta}} \int_{Y_{S}}^{Y_{N}} \frac{\partial \psi_{1}}{\partial y} q_{1}\right|_{x} d y \\
& \quad=\int_{Y_{S}}^{Y_{N}} \int_{X_{E}}^{x} w_{e} d x d y .
\end{aligned}
$$

Using Eq. (2), $\left.\psi_{1}\right|_{y=Y_{S}}=\left.\psi_{1}\right|_{y=Y_{N}}=0$ and the Sverdrup balance $\left(\partial \psi_{\mathrm{Sv}} / \partial x=w_{e}\right)$ and averaging in time, we obtained

$$
\begin{gathered}
-\frac{1}{\hat{\beta}}\left[\frac{1}{2}\left(\frac{\partial \bar{\psi}_{1}}{\partial y}\right)^{2}||_{x}^{Y_{N}}+\left.\frac{1}{\hat{\beta}} \int_{Y_{S}}^{Y_{N}} F_{I} \frac{H_{2}}{H_{0}} \frac{\partial \bar{\psi}_{1}}{\partial y}\left(\bar{\psi}_{1}-\bar{\psi}_{2}\right)\right|_{x} d y\right. \\
-\left.\frac{1}{\hat{\beta}} \int_{Y_{S}}^{Y_{N}} \overline{\frac{\partial \psi_{1}^{\prime}}{\partial y} q_{1}^{\prime}}\right|_{x} d y+\left.\int_{Y_{S}}^{Y_{N}} \bar{\psi}_{1}\right|_{x} d y=\int_{Y_{S}}^{Y_{N}} \hat{\psi}_{S v} d y,
\end{gathered}
$$

where overbars represent the time mean; $q_{1}^{\prime}$ and $\psi_{1}^{\prime}$ are defined as $q_{1}-\bar{q}_{1}$ and $\psi_{1}-\bar{\psi}_{1}$, respectively; and we assumed $\left(\partial^{2} / \partial x^{2}\right) \ll\left(\partial^{2} / \partial y^{2}\right)$ for $\bar{\psi}_{1}$. Because $\hat{\beta}$ is $O\left(10^{3}\right)$, if $\left[\left.0.5\left(\partial \bar{\psi}_{1} / \partial y\right)^{2}\right|_{x}\right]_{Y_{S}}^{Y_{N}},\left.\int_{Y_{S}}^{Y_{N}} F_{I}\left(H_{2} / H_{0}\right)\left(\partial \bar{\psi}_{1} / \partial y\right)\left(\bar{\psi}_{1}-\bar{\psi}_{2}\right)\right|_{x} d y$, and $\left.\int_{Y_{S}}^{Y_{N}} \overline{\left(\partial \psi_{1}^{\prime} / \partial y\right) q_{1}^{\prime}}\right|_{x} d y$ are $O(1)$, these terms can be neglected in comparison with $\int_{Y_{S}}^{Y_{N}} \hat{\psi}_{\mathrm{Sv}} d y$. If the mass transport through the longitude in question is zero, the second terms can be ignored $\left[O\left(10^{-2}\right)\right.$ compared to the fourth term on the left-hand side actually]. In the prematurely separated jet case, because $\bar{\psi}_{1} \approx \hat{\psi}_{\mathrm{Sv}}$ far from the jet, or $\partial \bar{\psi}_{1} / \partial y$ is of the same order as $d \hat{\psi}_{\mathrm{Sv}} / d y$ at the boundary, the first term on the left-hand side is sufficiently small $O\left(10^{-3}\right)$ and can be ignored. The third term is left, but the order was $O\left(10^{-2}\right)$ in all experiments of this study with a prematurely separated jet. Therefore, the three terms multiplied by $1 / \hat{\beta}$ are ignored and

$$
\int_{Y_{S}}^{Y_{N}} \bar{\psi}_{1} d y \approx \int_{Y_{S}}^{Y_{N}} \hat{\psi}_{\mathrm{Sv}} d y
$$

is obtained.

\section{REFERENCES}

Bleck, R., S. Dean, M. O'Keefe, and A. Sawdey, 1995: A comparison of data-parallel and message-passing versions of the Miami Isopycnic Coordinate Ocean Model. Parallel Comput., 21, 1695-1720, doi:10.1016/0167-8191(95)00043-3.

Bryan, K., and M. D. Cox, 1972: The circulation of the World Ocean: A numerical study. Part I, a homogeneous model. J. Phys. Oceanogr., 2, 319-335, doi:10.1175/1520-0485(1972)002<0319: TCOTWO $>2.0 . \mathrm{CO} ; 2$.

Cessi, P., and G. R. Ierley, 1995: Symmetry-breaking multiple equilibria in quasigeostrophic, wind-driven flows. J. Phys. Oceanogr., 25, 1196-1205, doi:10.1175/1520-0485(1995)025<1196: SBMEIQ>2.0.CO;2.

Dengo, J., 1993: The problem of Gulf Stream separation: A barotropic approach. J. Phys. Oceanogr., 23, 2182-2200, doi:10.1175/ 1520-0485(1993)023<2182:TPOGSS $>2.0 . C O ; 2$.

Fox-Kemper, B., 2005: Reevaluating the roles of eddies in multiple barotropic wind-driven gyres. J. Phys. Oceanogr., 35, 12631278, doi:10.1175/JPO2743.1.

Frankignoul, C., N. Sennéchael, Y.-O. Kwon, and M. A. Alexander, 2011: Influence of the meridional shifts of the Kuroshio and the 
Oyashio Extensions on the atmospheric circulation. J. Climate, 24, 762-777, doi:10.1175/2010JCLI3731.1.

Griffiths, R. W., and A. E. Kiss, 1999: Flow regimes in a wide 'slicedcylinder' model of homogeneous beta-plane circulation. J. Fluid Mech., 399, 205-236, doi:10.1017/S0022112099006370.

Haidvogel, D. B., J. C. McWilliams, and P. R. Gent, 1992: Boundary current separation in a quasigeostrophic eddy-resolving ocean model. J. Phys. Oceanogr., 22, 882-902, doi:10.1175/ 1520-0485(1992)022<0882:BCSIAQ > 2.0.CO;2.

Han, Y. J., 1984: A numerical world ocean general circulation model: Part II. A baroclinic experiment. Dyn. Atmos. Oceans, 8, 141-172, doi:10.1016/0377-0265(84)90020-4.

Ierley, G. R., 1987: On the onset of inertial recirculation in barotropic general circulation models. J. Phys. Oceanogr., 17, 2366-2374, doi:10.1175/1520-0485(1987)017<2366:OTOOIR >2.0.CO;2.

, 1990: Boundary layers in the general ocean circulation. Annu. Rev. Fluid Mech., 22, 111-142, doi:10.1146/annurev.fl.22.010190.000551.

— a nonlinear boundary-layer problem. Stud. Appl. Math., 75, $1-36$

— dominated flow in the wind-driven circulation. Part I: Slip. J. Mar. Res., 53, 703-737, doi:10.1357/0022240953213052.

Imai, Y., T. Aoki, and K. Takizawa, 2008: Conservative form of interpolated differential operator scheme for compressible and incompressible fluid dynamics. J. Comput. Phys., 227, 2263-2285, doi:10.1016/j.jcp.2007.11.031.

Jiang, S., F. Jin, and M. Ghil, 1995: Multiple equilibria, periodic, and aperiodic solutions in a wind-driven double-gyre, shallowwater model. J. Phys. Oceanogr., 25, 764-786, doi:10.1175/ 1520-0485(1995)025<0764:MEPAAS > 2.0.CO;2.

Kamenkovich, V. M., V. A. Sheremet, A. R. Pastushkov, and S. O. Belotserkovsky, 1995: Analysis of the barotropic model of the subtropical gyre in the ocean for finite Reynolds numbers. Part I. J. Mar. Res., 53, 959-994, doi:10.1357/0022240953212981.

Kiss, A. E., 2002: Potential vorticity "crises", adverse pressure gradients, and western boundary current separation. J. Mar. Res., 60, 779-803, doi:10.1357/002224002321505138.

Marshall, D. P., and D. P. Tansley, 2001: An implicit formula for boundary current separation. J. Phys. Oceanogr., 31, 1633-1638, doi:10.1175/1520-0485(2001)031<1633:AIFFBC > 2.0.CO;2.

McCalpin, J. D., and D. B. Haidvogel, 1996: Phenomenology of the low-frequency variability in a reduced-gravity, quasigeostrophic double-gyre model. J. Phys. Oceanogr., 26, 739-752, doi:10.1175/1520-0485(1996)026<0739:POTLFV > 2.0.CO;2.

McWilliams, J. C., 1996: Modeling the oceanic general circulation. Annu. Rev. Fluid Mech., 28, 215-248, doi:10.1146/ annurev.fl.28.010196.001243.
Milliff, R. F., J. Morzel, D. B. Chelton, and M. H. Freilich, 2004: Wind stress curl and wind stress divergence biases from rain effects on QSCAT surface wind retrievals. J. Atmos. Oceanic Technol., 21, 1216-1231, doi:10.1175/1520-0426(2004)021<1216: WSCAWS $>2.0 . \mathrm{CO} ; 2$.

Nakano, H., H. Tsujino, and R. Furue, 2008: The Kuroshio current system as a jet and twin "relative" recirculation gyres embedded in the Sverdrup circulation. Dyn. Atmos. Oceans, 45, 135-164, doi:10.1016/j.dynatmoce.2007.09.002.

Özgökmen, T. M., E. P. Chassignet, and A. M. Paiva, 1997: Impact of wind forcing, bottom topography, and inertia on midlatitude jet separation in a quasigeostrophic model. J. Phys. Oceanogr., 27, 2460-2476, doi:10.1175/1520-0485(1997)027<2460: IOWFBT $>2.0 . \mathrm{CO} ; 2$.

Pierini, S., 2006: A Kuroshio Extension system model study: Decadal chaotic self-sustained oscillations. J. Phys. Oceanogr., 36, 1605-1625, doi:10.1175/JPO2931.1.

Primeau, F. W., and D. Newman, 2007: Bifurcation structure of a wind-driven shallow water model with layer-outcropping. Ocean Modell., 16, 250-263, doi:10.1016/j.ocemod.2006.10.003.

Sasai, C. Y., K. J. Richards, A. Ishida, and H. Sasaki, 2010: Effects of cyclonic mesoscale eddies on the marine ecosystem in the Kuroshio Extension region using an eddy-resolving coupled physical-biological model. Ocean Dyn., 60, 693-704, doi:10.1007/ s10236-010-0264-8.

Sasaki, Y. N., and N. Schneider, 2011: Decadal shifts of the Kuroshio Extension jet: Application of thin-jet theory. J. Phys. Oceanogr., 41, 979-993, doi:10.1175/2010JPO4550.1.

Smith, R. D., M. E. Maltrud, F. O. Bryan, and M. W. Hecht, 2000: Numerical simulation of the North Atlantic Ocean at $1 / 10^{\circ}$. J. Phys. Oceanogr., 30, 1532-1561, doi:10.1175/ 1520-0485(2000)030<1532:NSOTNA $>2.0 . \mathrm{CO} ; 2$.

Sugimoto, S., and K. Hanawa, 2012: Relationship between the path of the Kuroshio in the south of Japan and the path of the Kuroshio Extension in the east. J. Oceanogr., 68, 219-225, doi:10.1007/s10872-011-0089-1.

Takano, K., Y. Mintz, and Y. Han, 1973: Numerical simulation of World Ocean circulation. Bull. Amer. Meteor. Soc., 54, 749.

Verron, J., and C. Le Provost, 1991: Response of eddy-resolved general circulation numerical models to asymmetrical wind forcing. Dyn. Atmos. Oceans, 15, 505-533, doi:10.1016/ 0377-0265(91)90002-W.

_ and J. H. Jo, 1994: On the stability of wind-driven barotropic ocean circulations. Fluid Dyn. Res., 14, 7-27, doi:10.1016/ 0169-5983(94)90019-1.

, and E. Blayo, 1996: The no-slip condition and separation of western boundary currents. J. Phys. Oceanogr., 26, 1938-1951, doi:10.1175/1520-0485(1996)026<1938:TNSCAS>2.0.CO;2. 